for conservation globally

Threatened

通
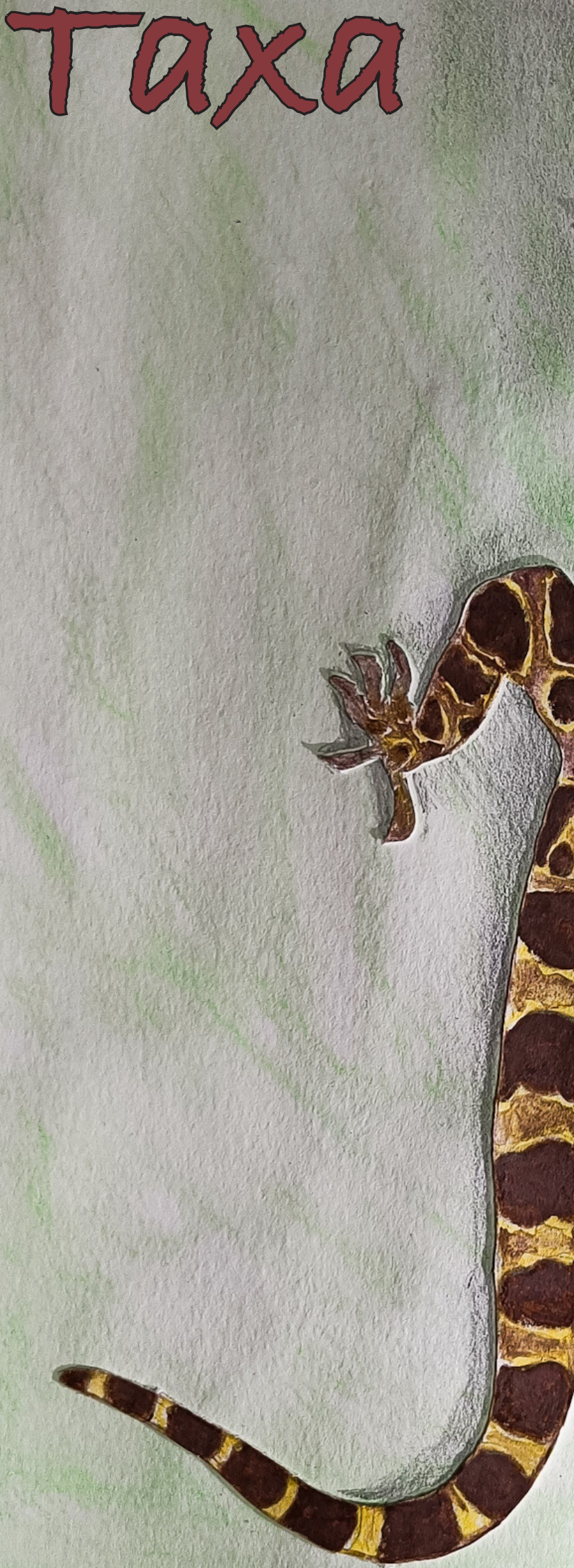

Open Access

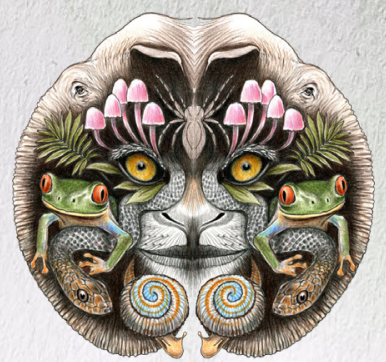

$10.1160 \mathrm{~g} / \mathrm{j}$ ott.2022.14.1.20311-20538 wWw.threatenedtaxa.org

26 January 2022 (Online \& Print) 14(1): 20311-20538 ISSN0974-7907 (Online) ISSN 0974-7893 (Print) 


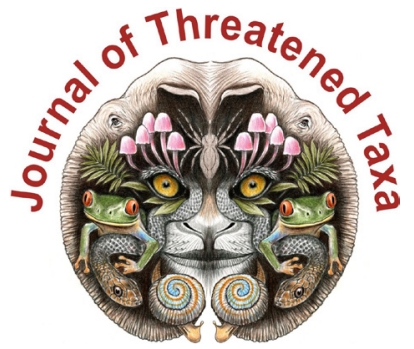

ISSN 0974-7907 (Online); ISSN $0974-7893$ (Print)

Publisher

Host

Wildlife Information Liaison Development Society

www.wild.zooreach.org

Zoo Outreach Organization www.zooreach.org

No. 12, Thiruvannamalai Nagar, Saravanampatti - Kalapatti Road, Saravanampatti, Coimbatore, Tamil Nadu 641035, India

Ph: +91 9385339863 | www.threatenedtaxa.org

Email: sanjay@threatenedtaxa.org

EDITORS

\section{Founder \& Chief Editor}

Dr. Sanjay Molur

Wildlife Information Liaison Development (WILD) Society \& Zoo Outreach Organization (ZOO),

12 Thiruvannamalai Nagar, Saravanampatti, Coimbatore, Tamil Nadu 641035, India

\section{Deputy Chief Editor}

Dr. Neelesh Dahanukar

Noida, Uttar Pradesh, India

\section{Managing Editor}

Mr. B. Ravichandran, WILD/ZOO, Coimbatore, India

\section{Associate Editors}

Dr. Mandar Paingankar, Government Science College Gadchiroli, Maharashtra 442605, India

Dr. Ulrike Streicher, Wildlife Veterinarian, Eugene, Oregon, USA

Ms. Priyanka Iyer, ZOO/WILD, Coimbatore, Tamil Nadu 641035, India

Dr. B.A. Daniel, ZOO/WILD, Coimbatore, Tamil Nadu 641035, India

\section{Editorial Board}

Dr. Russel Mittermeier

Executive Vice Chair, Conservation International, Arlington, Virginia 22202, USA

\section{Prof. Mewa Singh Ph.D., FASc, FNA, FNASc, FNAPsy}

Ramanna Fellow and Life-Long Distinguished Professor, Biopsychology Laboratory, and Institute of Excellence, University of Mysore, Mysuru, Karnataka 570006, India; Honorary Professor, Jawaharlal Nehru Centre for Advanced Scientific Research, Bangalore; and Adjunct Professor, National Institute of Advanced Studies, Bangalore

\section{Stephen D. Nash}

Scientific Illustrator, Conservation International, Dept. of Anatomical Sciences, Health Sciences Center, T-8, Room 045, Stony Brook University, Stony Brook, NY 11794-8081, USA

\section{Dr. Fred Pluthero}

Toronto, Canada

\section{Dr. Priya Davidar}

Sigur Nature Trust, Chadapatti, Mavinhalla PO, Nilgiris, Tamil Nadu 643223, India

\section{Dr. Martin Fisher}

Senior Associate Professor, Battcock Centre for Experimental Astrophysics, Cavendish

Laboratory, JJ Thomson Avenue, Cambridge CB3 OHE, UK

\section{Dr. John Fellowes}

Honorary Assistant Professor, The Kadoorie Institute, 8/F, T.T. Tsui Building, The University of Hong Kong, Pokfulam Road, Hong Kong

\section{Prof. Dr. Mirco Solé}

Universidade Estadual de Santa Cruz, Departamento de Ciências Biológicas, Vice-coordenado do Programa de Pós-Graduação em Zoologia, Rodovia Ilhéus/Itabuna, Km 16 (45662-000)

Salobrinho, Ilhéus - Bahia - Brasil

\section{Dr. Rajeev Raghavan}

Professor of Taxonomy, Kerala University of Fisheries \& Ocean Studies, Kochi, Kerala, India

\section{English Editors}

Mrs. Mira Bhojwani, Pune, India

Dr. Fred Pluthero, Toronto, Canad

Mr. P. Ilangovan, Chennai, India

Web Development

Mrs. Latha G. Ravikumar, ZOO/WILD, Coimbatore, India

\section{Typesetting}

Mr. Arul Jagadish, ZOO, Coimbatore, India

Mrs. Radhika, ZOO, Coimbatore, India

Mrs. Geetha, ZOO, Coimbatore India
Fundraising/Communications

Mrs. Payal B. Molur, Coimbatore, India

Subject Editors 2018-2020

Fungi

Dr. B. Shivaraju, Bengaluru, Karnataka, India

Dr. R.K. Verma, Tropical Forest Research Institute, Jabalpur, India

Dr. Vatsavaya S. Raju, Kakatiay University, Warangal, Andhra Pradesh, India

Dr. M. Krishnappa, Jnana Sahyadri, Kuvempu University, Shimoga, Karnataka, India

Dr. K.R. Sridhar, Mangalore University, Mangalagangotri, Mangalore, Karnataka, India

Dr. Gunjan Biswas, Vidyasagar University, Midnapore, West Bengal, India

\section{Plants}

Dr. G.P. Sinha, Botanical Survey of India, Allahabad, India

Dr. N.P. Balakrishnan, Ret. Joint Director, BSI, Coimbatore, India

Dr. Shonil Bhagwat, Open University and University of Oxford, UK

Prof. D.J. Bhat, Retd. Professor, Goa University, Goa, India

Dr. Ferdinando Boero, Università del Salento, Lecce, Italy

Dr. Dale R. Calder, Royal Ontaro Museum, Toronto, Ontario, Canada

Dr. Cleofas Cervancia, Univ. of Philippines Los Baños College Laguna, Philippines

Dr. F.B. Vincent Florens, University of Mauritius, Mauritius

Dr. Merlin Franco, Curtin University, Malaysia

Dr. V. Irudayaraj, St. Xavier's College, Palayamkottai, Tamil Nadu, India

Dr. B.S. Kholia, Botanical Survey of India, Gangtok, Sikkim, India

Dr. Pankaj Kumar, Kadoorie Farm and Botanic Garden Corporation, Hong Kong S.A.R., China

Dr. V. Sampath Kumar, Botanical Survey of India, Howrah, West Bengal, India

Dr. A.J. Solomon Raju, Andhra University, Visakhapatnam, India

Dr. Vijayasankar Raman, University of Mississippi, USA

Dr. B. Ravi Prasad Rao, Sri Krishnadevaraya University, Anantpur, India

Dr. K. Ravikumar, FRLHT, Bengaluru, Karnataka, India

Dr. Aparna Watve, Pune, Maharashtra, India

Dr. Qiang Liu, Xishuangbanna Tropical Botanical Garden, Yunnan, China

Dr. Noor Azhar Mohamed Shazili, Universiti Malaysia Terengganu, Kuala Terengganu, Malaysia

Dr. M.K. Vasudeva Rao, Shiv Ranjani Housing Society, Pune, Maharashtra, India

Prof. A.J. Solomon Raju, Andhra University, Visakhapatnam, India

Dr. Mandar Datar, Agharkar Research Institute, Pune, Maharashtra, India

Dr. M.K. Janarthanam, Goa University, Goa, India

Dr. K. Karthigeyan, Botanical Survey of India, India

Dr. Errol Vela, University of Montpellier, Montpellier, France

Dr. P. Lakshminarasimhan, Botanical Survey of India, Howrah, India

Dr. Larry R. Noblick, Montgomery Botanical Center, Miami, USA

Dr. K. Haridasan, Pallavur, Palakkad District, Kerala, India

Dr. Analinda Manila-Fajard, University of the Philippines Los Banos, Laguna, Philippines

Dr. P.A. Sinu, Central University of Kerala, Kasaragod, Kerala, India

Dr. Afroz Alam, Banasthali Vidyapith (accredited A grade by NAAC), Rajasthan, India

Dr. K.P. Rajesh, Zamorin's Guruvayurappan College, GA College PO, Kozhikode, Kerala, India

Dr. David E. Boufford, Harvard University Herbaria, Cambridge, MA 02138-2020, USA

Dr. Ritesh Kumar Choudhary, Agharkar Research Institute, Pune, Maharashtra, India

Dr. Navendu Page, Wildlife Institute of India, Chandrabani, Dehradun, Uttarakhand, India

\section{Invertebrates}

Dr. R.K. Avasthi, Rohtak University, Haryana, India

Dr. D.B. Bastawade, Maharashtra, India

Dr. Partha Pratim Bhattacharjee, Tripura University, Suryamaninagar, India

Dr. Kailash Chandra, Zoological Survey of India, Jabalpur, Madhya Pradesh, India

Dr. Ansie Dippenaar-Schoeman, University of Pretoria, Queenswood, South Africa

Dr. Rory Dow, National Museum of natural History Naturalis, The Netherlands

Dr. Brian Fisher, California Academy of Sciences, USA

Dr. Richard Gallon, llandudno, North Wales, LL30 1UP

Dr. Hemant V. Ghate, Modern College, Pune, India

Dr. M. Monwar Hossain, Jahangirnagar University, Dhaka, Bangladesh

Mr. Jatishwor Singh Irungbam, Biology Centre CAS, Branišovská, Czech Republic.

Dr. Ian J. Kitching, Natural History Museum, Cromwell Road, UK

Dr. George Mathew, Kerala Forest Research Institute, Peechi, India

For Focus, Scope, Aims, and Policies, visit https://threatenedtaxa.org/index.php/JoTT/aims_scope
For Article Submission Guidelines, visit https://threatenedtaxa.org/index.php/JoTT/about/submissions
For Policies against Scientific Misconduct, visit https://threatenedtaxa.org/index.php/JoTT/policies_various

continued on the back inside cover 


\title{
A floristic survey across three coniferous forests of Kashmir Himalaya, India - a checklist
}

\author{
Ashaq Ahmad Dar ${ }^{1}$ (D), Akhtar Hussain Malik ${ }^{2}$ iD $\&$ Narayanaswamy Parthasarathy ${ }^{3}$ (D) \\ ${ }^{1,3}$ Department of Ecology and Environmental Sciences, School of Life Sciences, Pondicherry University, Puducherry 605014, India \\ ${ }^{2}$ Centre for Biodiversity and Taxonomy, Department of Botany, University of Kashmir, Jammu \& Kashmir 190006, India \\ ${ }^{1}$ bscashaq@gmail.com, ${ }^{2}$ ecoakhtar@gmail.com, ${ }^{3}$ nparthasarathypu@gmail.com (corresponding author)
}

\begin{abstract}
This study presents a checklist of the flora of three coniferous forests of the Himalayan biodiversity hotspot in Kashmir: low-level blue pine (BP), mixed coniferous (MC) and subalpine (SA) forests. The list includes altitudinal distribution and conservation status of 272 vascular plant species representing 196 genera and 64 families. Excluding neophytes (70 taxa, 62 genera, and 27 families), Magnoliophyta comprised 190 taxa, 139 genera, and 50 families; Pinophyta seven taxa, six genera, and three families; and Pteridophyta three taxa, three genera, and two families. Most speciose families from Magnoliophyta include Compositae, Apiaceae, and Rosaceae. Genera such as Artemisia, Potentilla, Viola, and Saussurea contributed the maximum number of species. In case of Pinophyta, the principal families are Pinaceae with four taxa followed by Cupressaceae ( 2 taxa), whereas genus Juniperus comprised two species. In Pteridophyta, Pteridaceae ( 2 taxa) formed the most speciose family. The herbs contributed 177 taxa, followed by tress ( 15 taxa), shrubs ( 8 ) and subshrubs (2). The maximum number of taxa belongs to SA (136 taxa) followed by MC (134 taxa) and BP ( 83 taxa) forests. The species distribution reveals 20 , 30 , and 46 taxa are exclusive to BP, MC, and SA forests. More than $16 \%$ of taxa are categorized in the International Union for Conservation of Nature (IUCN) Red List, and 24 taxa are endemic to the Himalayan landscape. The checklist provides a roadmap for research, protection and conservation of plant diversity, especially the threatened taxa.
\end{abstract}

Keywords: Compositae, coniferous forest, conservation, elevation, floristic survey, hotspot, Kashmir Himalaya, mountains, threatened taxa.

Abbreviations: Afg.-Afghanistan | Ah-Annual herb | APG-Angiosperm Phylogeny Group | Bh-Biennial herb | BP-Low-level blue pine forest | C-Central | CBD-Convention on Biological Diversity | CR-Critically Endangered | DD-Data Deficient | DS-Deciduous shrub | DT-Deciduous tree | E-Eastern | EC- Eastern-central | EN-Endangered | ES-Evergreen shrub | ET-Evergreen tree | IHRIndian Himalayan Region | IUCN-International Union for Conservation of Nature | LC-Least Concern | MC-Mixed coniferous forest | Medit.-Mediterranean | Mya.-Myanmar | N-Northern | NA-Not assessed | NC-North-central | NE-North-eastern | NW-Northwestern | OER-Observed elevation range | Pak.-Pakistan | Ph-Perennial herb | Phip.-Philippines | S = Southern | S-Shrub | SASubalpine forest | SC-South-central | SE-South-eastern | SS-Subshrub | SW-South-western | Temp.-Temperate | Thail.-Thailand | TPL-The Plant List | VU-Vulnerable | W-Western.

Citation: Dar, A.A., A.H. Malik \& N. Parthasarathy (2022). A floristic survey across three coniferous forests of Kashmir Himalaya, India - a checklist. Journal of Threatened Taxa 14(1): 20323-20345. https://doi.org/10.11609/jott.7208.14.1.20323-20345

Copyright: @ Dar et al. 2022. Creative Commons Attribution 4.0 International License. JoTT allows unrestricted use, reproduction, and distribution of this article in any medium by providing adequate credit to the author(s) and the source of publication.

Funding: University Grant Commission (UGC) by means of Junior Research Fellowship (UGC-JRF) UGC-Ref. No.: 3796/(NET-JULY 2018).

Competing interests: The authors declare no competing interests.

Author details: ASHAQ AHMAD DAR is a research scholar/junior research fellow. His areas of interest are forest ecology and plant taxonomy. DR. AKHTAR HUSSAIN MALIK, junior scientist, works in the field of plant taxonomy, biodiversity, and ethnobotany. N. PARTHASARATHY, professor, has expertise in forest ecology, biodiversity conservation, and plant taxonomy.

Author contributions: AAD carried out the fieldwork, gathered, processed \& stored the specimens, and prepared the manuscript. AHM identified the plant specimens. NP directed the work and examined the manuscript.

Acknowledgements: Authors are indebted to the Jammu \& Kashmir Forest Department for kindly providing permission to carry out research fieldwork. We express gratitude to the Centre for Biodiversity and Taxonomy, Department of Botany, University of Kashmir for their paid assistance in identifying plant specimens. Field excursions would not have been conceivable without the enthusiastic assistance of Mudasir Ahmad Wani, Bilal Ahmad Dar, Basharat Ahmad Wani, Mushtaq Ahmad Dar, and many others especially guard officer Tariq Ahmad. Special thanks to Ayushi Kurian, Institut Français de Pondichéry, for GIS (map) related assistance.
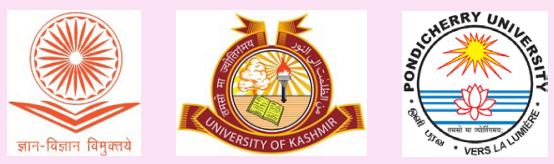


\section{INTRODUCTION}

Research on biodiversity became an essential aspect of biological research immediately after the Convention on Biological Diversity (CBD), with the goal of determining the implications of rapid depletion, management and climate change on species composition and diversity. Biodiversity-related data provide a foundation for species conservation and habitat protection (Cadotte 2006). With only $2.2 \%$ of global land area, India houses over 18,000 plant species, including 5,000 endemic flora, and is recognized among the 17 global mega-biodiverse countries (Nayar 1996; Singh et al. 2015). About half of the biodiversity hotspots representing $25 \%$ of the known biota are reported from mountain ecosystems (Wester et al. 2019). However, until recently, mountains acquired the attention of researchers, policy-makers, and conservationists.

Currently, diverse habitats supporting distinct flora are experiencing the threat of destruction due to fragmentation, rapid human population growth and climate change (Janssen et al. 2016; IUCN 2017). Consistent reductions in plant diversity call for continuous exploration of the population status of flora using systematic (IUCN) criteria, as this is acknowledged as the most rigorous strategy/technique for evaluating the global status of biodiversity and categorizing plants based on their projected risk of extinction (Maes et al. 2015; Orsenigo et al. 2018; Nowak et al. 2020).

The Himalaya, extending from Afghanistan to Myanmar, is one of 36 biodiversity hotspots harbouring a diverse range of flora and fauna, resulting from the phytogeographical complexity of the region (Zachos \& Habel 2011). About half of the known biodiversity in India, particularly endemics, is contributed by the $13 \%$ land area of the Indian Himalayan Region (IHR). The phytogeographical complexity in the present Jammu \& Kashmir, located on the northwestern side of the Himalaya, contributes significantly to various life forms. On account of its floristic status, the Kashmir Himalaya is a part of Himalayan biodiversity hotspot, and it is also considered to be vulnerable to climate change and thus species extinction (Rashid et al. 2015).

Several scholars over the course of time have made significant contributions to floristic knowledge of the Himalayan region: Hooker (1872-1897); Lambert (1933); Javeid (1966, 1978, 1979); Hajra (1983); Polunin \& Stainton (1984); Kachroo (1993); Singh \& Kachroo (1994); and Malik et al. (2010). However, critical taxonomic knowledge about the Kashmir Himalaya is still poor. In addition, a detailed study on the altitudinal distribution of taxa across the forest types is lacking. Consequently, the present study was undertaken to document the floristic diversity of the area, and to highlight its conservation significance.

\section{MATERIALS AND METHODS}

\section{Study area}

The study area spans over five districts of the Kashmir valley (33.513-34.659 ${ }^{0} \mathrm{~N}$ \& 74.497-75.019 ${ }^{0} \mathrm{E}$ ) in the present Jammu \& Kashmir, India (Figure 1; Image1). Kashmir valley exhibits a warm summer and humid continental climate (Dfa; Peel et al. 2007) with four distinctive seasons, i.e., spring, summer, autumn, and winter. Climate data from the last 38 years revealed that Kashmir valley experiences an annual mean minimum and maximum temperature of $5.4 \pm 0.4{ }^{\circ} \mathrm{C}$ and $17.6 \pm 0.8^{\circ} \mathrm{C}$ (Dad et al. 2021). Furthermore, the mean annual rainfall is $1005.5 \pm 197.6 \mathrm{~mm}$ (Dad et al. 2021). About $46 \%$ of precipitation occurs during pre-monsoon, followed by south-west monsoon (27\%), winter monsoon (25\%), and post-monsoon (8\%). Disturbances posed by the Mediterranean Sea during winter lead to frequent rain and snowfall in the valley. The period of snowfall extends from October-March. Geologically, the study area consists of rocks chiefly composed of slates, phyllites and quartzites (Krishnan 1982). The predominant soil orders are entisols, inceptisols, alfisols, and mollisols (Mahapatra et al. 2000; Sidhu \& Surya 2014).

Low-level blue pine (BP) forest ranges from 1,500$2,400 \mathrm{~m}$ on gentle to moderate slopes. Even-aged stands of the blue-pine, Pinus wallichiana A.B.Jacks intermixed with deodar, Cedrus deodara (Roxb. ex D.Don) G.Don and the spruce, Picea smithiana (Wall.) Boiss., occur depending upon the aspect. Since the ground surface is covered with litter, understorey herb vegetation is less comprising of Poa alpina L., Fragaria nubicola (Lindl. ex Hook.f.) Lacaita, Viola canescens Wall. in summer season (Shaheen et al. 2012). Dominant shrub species include Viburnum grandiflorum Wall. ex DC., Berberis lycium Royle, Indigofera heterantha Brandis depending upon aspect and canopy cover. Anthropogenic disturbances include land encroachment (for cultivating Zea mays L. and Solanum tuberosum L.), non-timber forest product extraction (fruits of Viburnum grandiflorum Wall. ex DC., medicinally important herbs, honey, nutritious and medicinally important fungus - Morchella esculenta (L.) Pers. etc.), lopping, firewood collection, grazing, and fire.

Mixed coniferous (MC) forest, commonly referred to 


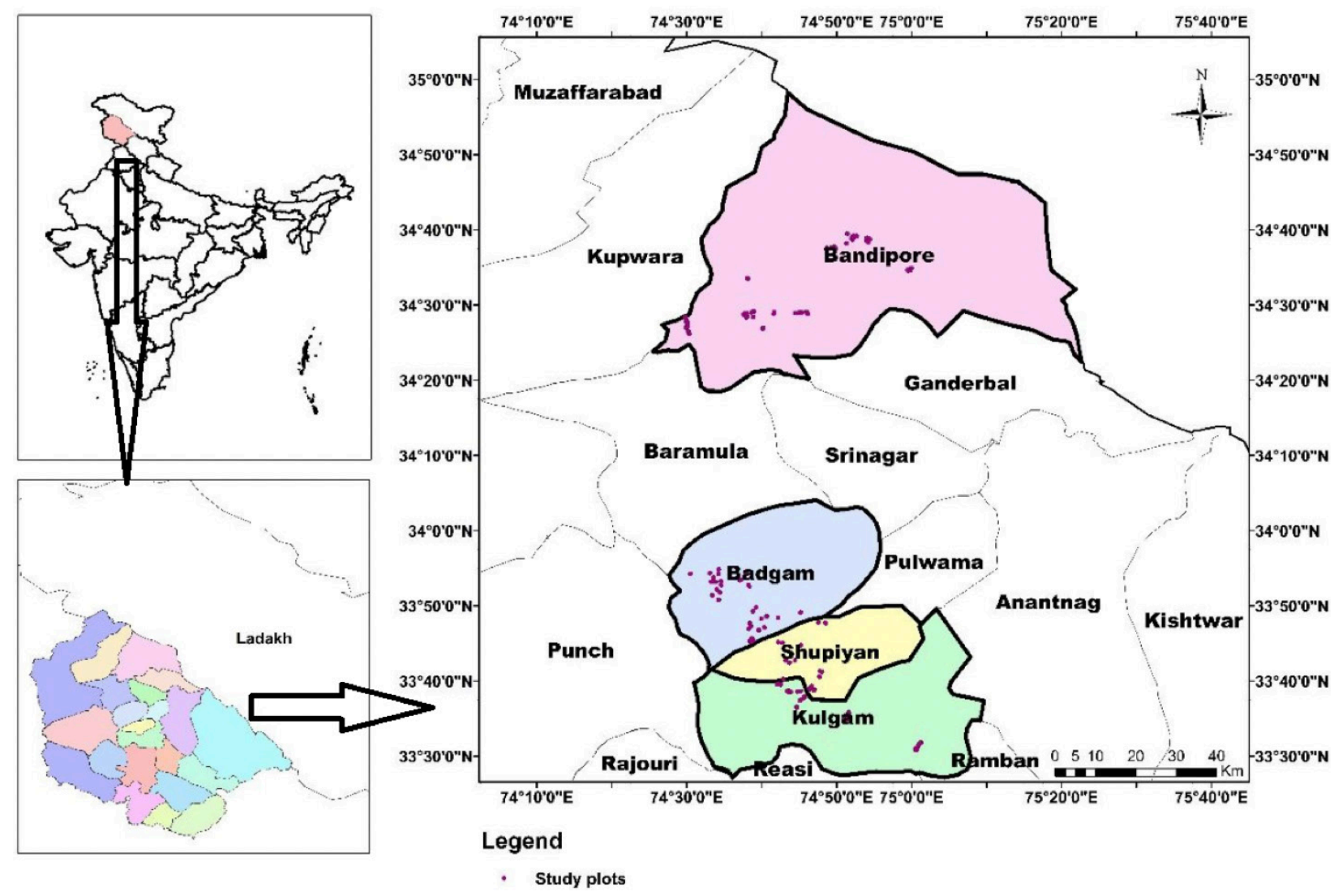

Table 1. Map of India, Jammu and Kashmir state, four districts shown in different colours, and the sampled locations in red dots.

as fir forest, occupies the central and western Himalaya from an elevation of about 2,400-3,000 m. Tree species such as evergreen coniferous (Abies pindrow (Royle ex D.Don) Royle, Picea smithiana and Pinus wallichiana) and deciduous broad-leaved tree species (Acer caesium Wall. ex Brandis, and Prunus cornuta (Wall. ex Royle) Steud.,) predominate. The regeneration of tree species is low or absent, as indicated by the presence of few saplings and seedlings. Understorey vegetation blossoms after the snowmelt during the spring season and is quite dense and diverse. The dominant shrub and herb species include Viburnum grandiflorum and Stipa sibirica (L.) Lam., (Dar \& Sundarapandian 2016). Epiphytic moss and lichen cover the trunk and lower branches of emergent tree species. Activities such as grazing, extraction of plants and plant materials of economic and medicinal value, firewood collection, illegal logging, etc., contribute to forest degradation.

The subalpine forest (SA) forms a transition between $M C$ forest and alpine scrub or grassland from 2,900-3,500 m. Abies pindrow is a characteristic and dominant species intermixed with Betula utilis D.Don. Rhododendron spp. occur as undergrowth or form individual stands. The species of Primulaceae, Ranunculaceae, and Compositae constitute the main understory herbaceous vegetation. The subalpine forest is equally subjected to anthropogenic disturbances like the other forest types besides heavy winter snowfall as a natural disturbance (Gairola et al. 2009).

\section{Sampling, herbarium preparation, and data analysis}

A reconnaissance floristic survey was undertaken in the landscape between the elevation gradient of $1,500 \mathrm{~m}$ and $3,800 \mathrm{~m}$ to understand the forest types and composition. Three coniferous forests of Kashmir Himalaya: BP, MC, and SA (Champion \& Seth 1968) were identitifed in the region. Botanical explorations were undertaken during 2019 (March-July) and 2020 (MayAugust) by employing a random sampling approach considering the accessability and forest types. During the survey, plants such as trees, shrubs and herbs were documented and voucher specimens were collected. Specimens were processed (pressing, drying, chemical treatment, and mounting) following recommended standard techniques (Rao \& Sharma 1990), and examined and identified at the Centre for Biodiversity 
and Taxonomy, University of Kashmir. The voucher specimens were deposited at the Department of Ecology and Environmental Sciences Herbarium, Pondicherry University. The Plant List (TPL; http://www.theplantlist. org/) was referred for updated binomial nomenclature and the author names. Angiosperm Phylogeny Group III (APG III) Classification (2009) and Chase \& Reveal (2009) for angiosperms and Gymnosperms were followed for categorizing families. Khuroo et al. (2007) was referred for the origin and alien status of flora. Various information sources were explored to acquire Himalayan and global records of inventoried taxa, including Himalayan flora literature (Hooker 1872-1897; Polunin \& Stainton 1984), Tropicos (http://www.tropicos.org/), India Biodiversity Portal (https://indiabiodiversity.org/), Flowers of India (http://www.flowersofindia.net/) and Plants of the World online (http://www.plantsoftheworldonline. org/).

\section{RESULTS}

\section{Species composition and distribution}

A total of 272 taxa belonging to 196 genera and 64 families were recorded across the three Kashmir Himalayan coniferous forests (Table 1). Of the total vascular plants, neophytes (aliens) represent 70 (25.73\%) taxa within 27 and 62 families and genera (Table 2). This includes invasive aliens (IA; 51.42\%), naturalised aliens (NZ; 38.57\%), casual/naturalised aliens (C/NA; 8.57\%) and cultivated unescaped aliens (CU; $1.43 \%$ ). Among the aliens, woody flora accounted five $(7.14 \%)$ species (Robinia pseudoacacia L., Syringa emodi Wall. ex Royle, Crataegus songarica K. Koch, Rosa brunonii Lindl., Aesculus indica (Wall. ex Cambess.) Hook.). All the neophytes are excluded hereafter from further analysis.

Most of the native taxa belong to Magnoliophyta (192 taxa, 139 genera, and 50 families), whereas Pinophyta (seven taxa, six genera, and three families) and Pteridophyta (three taxa, three genera, and two families) are less represented (Table 2). Within Magnoliophyta, 177 taxa (92\%) belong to Magnoliopsida and $15(7.8 \%)$ to Liliopsida. Among these, there are 177 herb taxa (174 Magnoliophyta and three Pteridophyta), eight shrub taxa (Magnoliophyta only), 15 tree taxa (eight Magnoliophyta and seven Pinophyta) and two subshrubs (Magnoliophyta only). Herbs are dominated by perennials (150 taxa, 85\%), followed by annuals (17 taxa, 9.6\%), biennials (two taxa, 1.1\%) and evergreen (one taxon, $0.56 \%$ ). Moreover, seven (3.9\%) herbaceous taxa are either perennials, annuals or biennials (Table 2). Of the 15 reported tree taxa, most of them are deciduous (8, 59\%), followed by evergreen conifers (seven, $41 \%)$. Similarly, among the shrubs, seven (88\%) are deciduous (including one climber), and one (12.5\%) is evergreen. The images of selected plant taxa are provided (Images 2-7).

Three families in Magnoliophyta with greater contribution to species richness include Compositae (28 taxa, 13.86\%) and Apiaceae and Rosaceae (13, 6.44\% each). Families with ten or more species (besides above three) include Lamiaceae, Leguminosae, Poaceae (11, $5.45 \%$ each), and Ranunculaceae (10, 4.95\%) (Figure 2). Species-rich genera, i.e., Artemisia, Potentilla, Viola, and Saussurea contributed 16 (7.92\%) taxa. Majority of families $(26,47.27 \%)$ and genera $(108,72.97 \%)$ are monotypic with a single taxon. Among Pinophyta, Pinaceae (four taxa) and Cupressaceae (two taxa) are predominant families, whereas Juniperus is the principal genus contributing two taxa. Pteridophyta is represented by Pteridaceae (two taxa) and Equisetaceae (one taxon), and all the three genera (Adiantum, Equisetum, and Pteris) contributed equally, i.e., one species. In contrast to tree and understory herb vegetation, all shrub families and genera contributed one species each.

The number of taxa varied among the forest types and corresponding elevation due to the uneven distribution of taxa (Table 1). The SA and MC forests represent greater number of taxa, i.e., 136 and 134, followed by BP forest ( 83 taxa). The species distribution revealed that 20 taxa are exclusive to BP forest, whereas 30 and 46 taxa are limited to $\mathrm{MC}$ and SA forests. However, $22.77 \%$ of taxa with a wide distributional range are shared among forest types. Furthermore, BP \& MC, BP \& SA, and MC

Table 1. Distribution of taxa among various taxonomic groups in three coniferous forests viz., low-level blue pine forest (BP), mixed coniferous forest (MC), subalpine forest (SA) of Kashmir Himalaya, India.

\begin{tabular}{|l|c|c|c|c|c|c|c|}
\hline Phylum & Taxon & Genera & Family & Trees & Shrubs & Subshrub & Herbs \\
\hline Magnoliophyta & 262 & 187 & 59 & 10 & 10 & 3 & 239 \\
\hline Pinophyta & 7 & 6 & 3 & 7 & - & - & - \\
\hline Pteridophyta & 3 & 3 & 2 & - & - & - & 3 \\
\hline Total & 272 & 196 & 64 & 17 & 10 & 3 & 242 \\
\hline
\end{tabular}




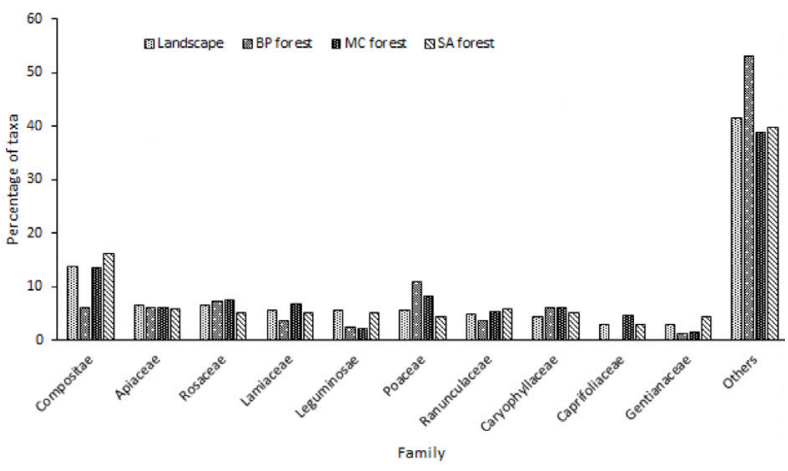

Figure 2. Percentage contribution to taxa across three coniferous forests of Kashmir Himalaya, India by families.

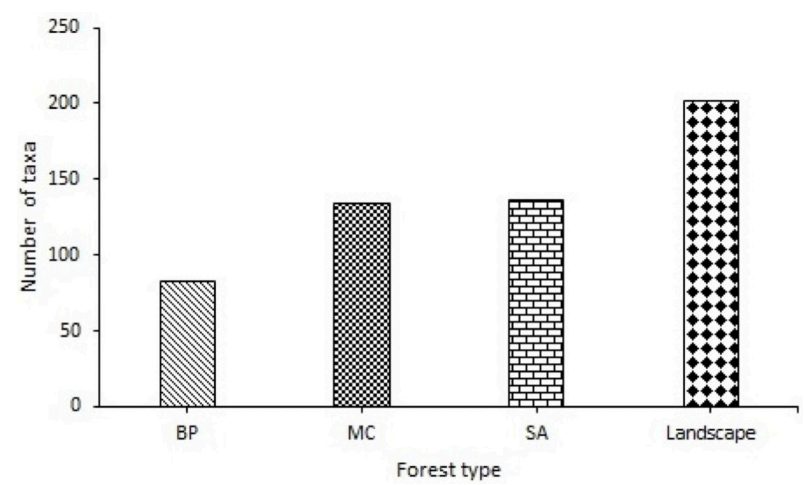

Figure 3. Number of taxa in three coniferous forests of Kashmir Himalaya, India.

\& SA forests shared 16, two, and 43 taxa, respectively. The SA forest harbours greater number of species of Compositae (16.18\%) and Caryophyllaceae (5.15\%) than to landscape-scale flora (13.86\% and $4.46 \%)$ in top 10 families. Similarly, Poaceae, and Rosaceae in BP $(10.84 \%$ \& $7.23 \%$ ) and $M C$ forests (8.21\% \& 7.46\%) contributed greater number of taxa than to the overall landscape (5.45\% \& 6.44\%).

\section{Determination of phytogeographic distribution and taxa status}

The distribution of most of the recorded taxa is confined to the northern temperate regions. However, 24 taxa restricted their distribution to the Himalayan landscape (Table 2). Despite the considerable research on plant conservation in Kashmir Himalaya, the analysis of the conservation status of the flora revealed that 169 taxa are not assessed (NA), and the remaining $33(16.37 \%)$ taxa are included under IUCN Red List category (Table 2). Among them, two species Saussurea costus (Falc.) Lipsch. and Aconitum chasmanthum Stapf ex Holmes are Critically Endangered (CR); four

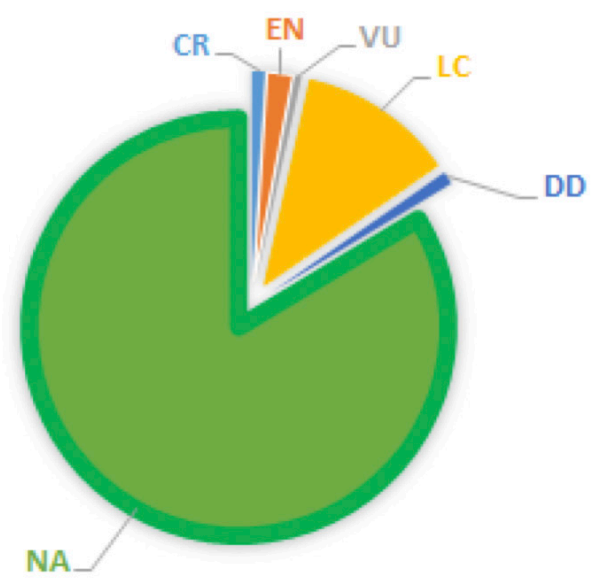

Figure 4. Pie-chart showing IUCN category of species across the three coniferous forests of Kashmir Himalaya, India.

species Trillium govanianum Wall. ex D.Don, Aconitum heterophyllum Wall. ex Royle, Taxus wallichiana Zucc. and Atropa acuminata Royle ex Lindl. are Endangered (EN); one species Cypripedium cordigerum D.Don is Vulnerable (VU), two species Asparagus filicinus Buch.Ham. ex D.Don and Corylus jacquemontii Decne. fall under Data Deficient (DD) category and 24 species are Least Concern (LC). With regard to the forest type and vertical distribution, the maximum number of threatened taxa (VU+EN+CR) occur in SA forest at high altitudinal zones.

\section{DISCUSSION}

The floristic survey revealed 272 taxa from 196 genera and 64 families categorized in three life-forms, i.e., trees and understorey shrubs and herbs (Table 1 \& 2). The number of taxa reported in the present study was greater than most of the floristic studies in temperate Kashmir Himalaya (Shaheen et al. 2012; Mir et al. 2019; Malik et al. 2021) and other Himalayan studies (Ahmad et al. 2020; Asif et al. 2020; Tiwari et al. 2020) and also elsewhere (Bai et al. 2011). Compositae and Apiaceae constituted species-rich families in this survey. These families were also well represented in other studies of the Kashmir Himalaya: Asif et al. (2020) Betula forests in northwestern Kashmir Himalaya; Dar \& Sundarapandian (2016) forests of western Himalaya, and elsewhere Devi et al. (2014) northwestern Himalaya. Variation in species distribution among the forest types/altitudinal zones could be due to micro-climatic heterogeneity resulting from a change in elevation, slope, and other ecological gradients (Körner 2007), besides evolutionary 
Table 2. List of plant species in three temperate coniferous forests, viz., low-level blue pine forest (BP), mixed coniferous forest (MC), subalpine forest (SA) of Kashmir Himalaya, India.

\begin{tabular}{|c|c|c|c|c|c|}
\hline Family/Taxon & Life-form & Forest type & OER & Voucher no. & Phytogeographic distribution \\
\hline \multicolumn{6}{|l|}{ Acanthaceae } \\
\hline Pteracanthus alatus (Nees) Bremek. ${ }^{1}$ & Erect $\mathrm{S}$ & BP & $2200-2300$ & $\mathrm{PU} / \mathrm{EES} / \mathrm{KH}-1210$ & $\begin{array}{l}\text { E. Afg. to S. China, N. Indo- } \\
\text { China \& Taiwan }\end{array}$ \\
\hline \multicolumn{6}{|l|}{ Adoxaceae } \\
\hline Sambucus wightiana Wall. ex Wight \& Arn. ${ }^{1^{*}}$ & Erect Ph & $\mathrm{BP} / \mathrm{MC} / \mathrm{SA}$ & $2200-3310$ & PU/EES/KH-15201 & India, Pak., W. Himalayas \\
\hline Viburnum grandiflorum Wall. ex DC. ${ }^{1}$ & DS & $\mathrm{BP} / \mathrm{MC} / \mathrm{SA}$ & $1890-3000$ & $\mathrm{PU} / \mathrm{EES} / \mathrm{KH}-1206$ & $\begin{array}{l}\text { Himalayas from Kashmir to } \\
\text { SE Tibet }\end{array}$ \\
\hline \multicolumn{6}{|l|}{ Amaranthaceae } \\
\hline Achyranthes aspera $\mathrm{L}^{.{ }^{*}}$ & $\mathrm{Ph}$ & $\mathrm{MC} / \mathrm{SA}$ & $2600-3000$ & $\mathrm{PU} / \mathrm{EES} / \mathrm{KH}-15001$ & $\begin{array}{l}\text { Tropical \& Subtropical Old } \\
\text { World; throughout India }\end{array}$ \\
\hline Chenopodium album L..* & Ah & $\mathrm{MC} / \mathrm{SA}$ & $2650-2990$ & PU/EES/KH-15065 & $\begin{array}{l}\text { Temp. Eurasia to Indian } \\
\text { Subcontinent }\end{array}$ \\
\hline Chenopodium foliosum Asch..$^{*}$ & Ah or Ph & SA & $2910-3160$ & PU/EES/KH-15066 & $\begin{array}{l}\text { C. \& S. Europe to Nepal; W. } \\
\text { Himalayas in India }\end{array}$ \\
\hline \multicolumn{6}{|l|}{ Amaryllidaceae } \\
\hline Allium humile Kunth ${ }^{1}$ & Bulbous Ph & $\mathrm{MC} / \mathrm{SA}$ & 2700-3015 & PU/EES/KH-15013 & $\begin{array}{l}\text { N. Pak. to C. Himalayas \& } \\
\text { China }\end{array}$ \\
\hline \multicolumn{6}{|l|}{ Apiaceae } \\
\hline Aegopodium alpestre Ledeb. $^{1}$ & $\mathrm{Ph}$ & MC & $2500-2600$ & $\mathrm{PU} / \mathrm{EES} / \mathrm{KH}-15008$ & $\begin{array}{l}\text { Temp. Asia; W. Himalayas } \\
\text { in India }\end{array}$ \\
\hline $\begin{array}{l}\text { Bunium cylindricum (Boiss. \& Hohen.) } \\
\text { Drude }^{1}\end{array}$ & $\mathrm{Ph}$ & SA & $3100-3150$ & PU/EES/KH-15045 & $\begin{array}{l}\text { Turkey to C. Asia \& Pak. to W. } \\
\text { Himalayas }\end{array}$ \\
\hline Bupleurum falcatum L. ${ }^{1}$ & $\mathrm{Ph}$ & BP & $2200-2300$ & PU/EES/KH-15046 & Europe to Himalayas \\
\hline Bupleurum longicaule Wall. ex DC. ${ }^{1}$ & $\mathrm{Ph}$ & SA & $3750-3800$ & $\mathrm{PU} / \mathrm{EES} / \mathrm{KH}-15047$ & $\begin{array}{l}\text { Himalayas from Pak. to } \\
\text { Bhutan }\end{array}$ \\
\hline Carum carvi L. ${ }^{1}$ & $\mathrm{Ph}$ & $\mathrm{BP}$ & $2350-2400$ & PU/EES/KH-15054 & $\begin{array}{l}\text { Palearctic region; throughout } \\
\text { India }\end{array}$ \\
\hline Chaerophyllum reflexum Aitch. ${ }^{1}$ & $\mathrm{Ph}$ & $\mathrm{BP} / \mathrm{MC}$ & $1927-2450$ & PU/EES/KH-15063 & $\begin{array}{l}\text { Himalayas from Pak. to SW } \\
\text { China }\end{array}$ \\
\hline Chaerophyllum villosum Wall. ex DC. ${ }^{1}$ & $\mathrm{Ph}$ & $\mathrm{BP} / \mathrm{MC} / \mathrm{SA}$ & $2050-2920$ & PU/EES/KH-15064 & $\begin{array}{l}\text { N. Pak. to China; Himalayas } \\
\text { in India }\end{array}$ \\
\hline Eryngium billardieri Delile ${ }^{1^{*}}$ & $\mathrm{Ph}$ & $\mathrm{BP}$ & $2120-2130$ & PU/EES/KH-15103 & $\begin{array}{l}\text { EC Turkey to Lebanon \& W. } \\
\text { Pak.; W. Himalayas in India }\end{array}$ \\
\hline Heracleum candicans Wall. ex DC. ${ }^{1}$ & Climbing Ph & $\mathrm{MC} / \mathrm{SA}$ & $2400-3810$ & PU/EES/KH-15119 & $\begin{array}{l}\text { Himalayas from Pak. to SW } \\
\text { China }\end{array}$ \\
\hline Pimpinella acuminata (Edgew.) C.B. Clarke ${ }^{1}$ & $\mathrm{Ph}$ & $\mathrm{BP} / \mathrm{MC} / \mathrm{SA}$ & $2200-3120$ & PU/EES/KH-15170 & $\begin{array}{l}\text { N. Pak. to China; Himalayas } \\
\text { in India }\end{array}$ \\
\hline Pimpinella diversifolia DC. ${ }^{1}$ & $\mathrm{Ph}$ & $\mathrm{MC}$ & $2460-2770$ & PU/EES/KH-15171 & $\begin{array}{l}\text { E. Afg. to China \& Indo-China; } \\
\text { Himalayas in India }\end{array}$ \\
\hline Prangos pabularia Lindl. $^{1}$ & $\mathrm{Ph}$ & $\mathrm{MC} / \mathrm{SA}$ & $2720-3140$ & PU/EES/KH-15189 & $\begin{array}{l}\text { Turkey to C. Asia \& W. } \\
\text { Himalayas }\end{array}$ \\
\hline Sanicula elata Buch.-Ham. ex D.Don ${ }^{1}$ & $\mathrm{Ph}$ & SA & $2910-2930$ & PU/EES/KH-15202 & $\begin{array}{l}\text { SE Asia from Pak. to W. China } \\
\text { \& S. Japan to SE Africa }\end{array}$ \\
\hline Scandix pecten-veneris L.. ${ }^{*}$ & Tall robust $\mathrm{Ph}$ & SA & $3300-3310$ & PU/EES/KH-15207 & Europe to NW India \\
\hline Selinum vaginatum C.B. Clarke ${ }^{1}$ & $\mathrm{Ph}$ & SA & $3790-3800$ & $\mathrm{PU} / \mathrm{EES} / \mathrm{KH}-15211$ & NE Pak. to W. Himalayas \\
\hline Seseli libanotis (L.) W.D.J.Koch ${ }^{1}$ & $\mathrm{Ph}$ & $\mathrm{MC} / \mathrm{SA}$ & $2740-2920$ & PU/EES/KH-15214 & $\begin{array}{l}\text { Europe, Turkey, Iran, W. Pak. } \\
\text { \& India }\end{array}$ \\
\hline \multicolumn{6}{|l|}{ Apocynaceae } \\
\hline Vincetoxicum hirundinaria Medik. ${ }^{1}$ & $\begin{array}{l}\text { Prostrate erect or } \\
\text { climbing Ah }\end{array}$ & $\mathrm{BP} / \mathrm{MC}$ & $1980-2760$ & PU/EES/KH-15244 & $\begin{array}{l}\text { Europe to W. Siberia \& N. } \\
\text { Turkey, NW Africa, Himalayas }\end{array}$ \\
\hline \multicolumn{6}{|l|}{ Araceae } \\
\hline Arisaema jacquemontii Blume ${ }^{2}$ & Rhizomatous Ph & $\mathrm{BP} / \mathrm{MC} / \mathrm{SA}$ & $2250-2950$ & PU/EES/KH-15028 & Afg. to Mya. \\
\hline Arisaema propinquum Schott $^{1}$ & $\mathrm{Ph}$ & $\mathrm{MC} / \mathrm{SA}$ & $2450-2950$ & PU/EES/KH-15029 & Pak. to Himalayas \& Tibet \\
\hline \multicolumn{6}{|l|}{ Araliaceae } \\
\hline Hedera nepalensis K.Koch ${ }^{1}$ & $\mathrm{Ph}$ & $\mathrm{BP} / \mathrm{MC}$ & $1980-2610$ & PU/EES/KH-15118 & $\begin{array}{l}\text { Afg. to Thail.; Himalayas in } \\
\text { India }\end{array}$ \\
\hline
\end{tabular}




\begin{tabular}{|c|c|c|c|c|c|}
\hline Family/Taxon & Life-form & Forest type & OER & Voucher no. & Phytogeographic distribution \\
\hline \multicolumn{6}{|l|}{ Asparagaceae } \\
\hline Asparagus filicinus Buch.-Ham. ex D.Don ${ }^{4}$ & $\begin{array}{l}\text { Erect or twining } \\
\text { Ph }\end{array}$ & BP & $1800-1900$ & PU/EES/KH-15036 & Himalayas to C. China \\
\hline Polygonatum multiflorum (L.) All. ${ }^{1}$ & Tufted Ah & $\mathrm{BP} / \mathrm{MC}$ & $2270-2440$ & PU/EES/KH-15181 & Eurasia; W. Himalayas in India \\
\hline Polygonatum verticillatum (L.) All. ${ }^{1}$ & Rhizomatous Ph & $\mathrm{BP} / \mathrm{MC} / \mathrm{SA}$ & $1980-3120$ & $\mathrm{PU} / \mathrm{EES} / \mathrm{KH}-15183$ & $\begin{array}{l}\text { Europe to China; Himalayas } \\
\text { in India }\end{array}$ \\
\hline \multicolumn{6}{|l|}{ Balsaminaceae } \\
\hline Impatiens brachycentra Kar. \& Kir. ${ }^{1}$ & $\mathrm{Ph}$ & $\mathrm{BP} / \mathrm{MC} / \mathrm{SA}$ & $2120-3310$ & PU/EES/KH-15122 & Afg. to C. Asia \& W. Himalayas \\
\hline \multicolumn{6}{|l|}{ Berberidaceae } \\
\hline Berberis lycium Royle $^{1}$ & Semi-DS & $\mathrm{BP}$ & $2100-2150$ & $\mathrm{PU} / \mathrm{EES} / \mathrm{KH}-1203$ & $\begin{array}{l}\text { W. Himalayas from Pak. to } \\
\text { Nepal }\end{array}$ \\
\hline Epimedium elatum C.Morren \& Decne. ${ }^{1}$ & Rhizomatous Ph & $\mathrm{MC} / \mathrm{SA}$ & $2520-3120$ & PU/EES/KH-15095 & N. Pak. to W. Himalayas \\
\hline Podophyllum hexandrum Royle $^{1}$ & Rhizomatous Ph & $\mathrm{BP} / \mathrm{MC} / \mathrm{SA}$ & $2370-3310$ & PU/EES/KH-15176 & $\begin{array}{l}\text { NE Afg. to C. China; Himalayas } \\
\text { in India }\end{array}$ \\
\hline \multicolumn{6}{|l|}{ Betulaceae } \\
\hline Betula utilis D.Don ${ }^{2}$ & DT & SA & $2910-3300$ & $\mathrm{PU} / \mathrm{EES} / \mathrm{KH}-1004$ & $\begin{array}{l}\text { Afg. to N. \& C. China; } \\
\text { Himalayas in India }\end{array}$ \\
\hline Corylus jacquemontii Decne. ${ }^{4}$ & DT & MC & $2560-2790$ & $\mathrm{PU} / \mathrm{EES} / \mathrm{KH}-1006$ & $\begin{array}{l}\text { Europe, Himalayas from Afg. } \\
\text { to W. Nepal }\end{array}$ \\
\hline \multicolumn{6}{|l|}{ Boraginaceae } \\
\hline $\begin{array}{l}\text { Arnebia benthamii (Wall. ex G. Don) I.M. } \\
\text { Johnst. }^{1}\end{array}$ & Rhizomatous Ph & SA & $3800-3900$ & $\mathrm{PU} / \mathrm{EES} / \mathrm{KH}-15024$ & $\begin{array}{l}\text { NE Pakistan to W. \& C. } \\
\text { Himalaya }\end{array}$ \\
\hline Cynoglossum glochidiatum Wall. ex Benth. ${ }^{1}$ & $\mathrm{Bh}$ & $\mathrm{BP} / \mathrm{MC} / \mathrm{SA}$ & $2120-3000$ & PU/EES/KH-15084 & $\begin{array}{l}\text { Afg. through Kashmir to } \\
\text { Sikkim \& W. China }\end{array}$ \\
\hline Cynoglossum lanceolatum Forssk. ${ }^{1^{*}}$ & $\mathrm{Bh}$ or $\mathrm{Ph}$ & $\mathrm{BP} / \mathrm{SA}$ & $2230-3800$ & PU/EES/KH-15085 & $\begin{array}{l}\text { Tropical \& S. Africa to } \\
\text { Tropical \& Subtropical Asia; } \\
\text { throughout India }\end{array}$ \\
\hline Hackelia uncinata (Benth.) C.E.C.Fisch ${ }^{1}$ & $\mathrm{Ph}$ & SA & $2910-3120$ & $\mathrm{PU} / \mathrm{EES} / \mathrm{KH}-15117$ & $\begin{array}{l}\text { Himalayas from Pak. to SW } \\
\text { China }\end{array}$ \\
\hline Myosotis alpestris F.W. Schmidt ${ }^{1}$ & $\mathrm{Ph}$ & SA & $3150-3310$ & PU/EES/KH-15148 & $\begin{array}{l}\text { Europe, Himalayas from Pak. } \\
\text { to Bhutan }\end{array}$ \\
\hline Myosotis sylvatica Ehrh. ex Hoffm. ${ }^{1}$ & $\mathrm{Ph}$ & $\mathrm{BP} / \mathrm{MC} / \mathrm{SA}$ & $2260-3150$ & PU/EES/KH-15149 & $\begin{array}{l}\text { Temp. Eurasia; W. Himalayas } \\
\text { in India }\end{array}$ \\
\hline \multicolumn{6}{|l|}{ Brassicaceae } \\
\hline Arabis amplexicaulis Edgew. ${ }^{1}$ & $\mathrm{Ph}$ & $\mathrm{BP} / \mathrm{MC}$ & $2200-2410$ & PU/EES/KH-15021 & Afg. to Mongolia \& Himalayas \\
\hline Arabis pterosperma Edgew. ${ }^{1}$ & $\mathrm{Ph}$ & MC & $2700-2800$ & PU/EES/KH-15023 & Kashmir to China \\
\hline Capsella bursa-pastoris (L.) Medik. ${ }^{1^{*}}$ & Erect $\mathrm{Ah}$ or $\mathrm{Bh}$ & $\mathrm{MC} / \mathrm{SA}$ & $2420-2950$ & PU/EES/KH-15053 & $\begin{array}{l}\text { Temp. Eurasia, N. Africa; } \\
\text { throughout India }\end{array}$ \\
\hline Chorispora tenella (Pall.) DC. ${ }^{1}$ & Ah & MC & $2750-2770$ & PU/EES/KH-15067 & $\begin{array}{l}\text { SE \& E. Europe to China; W. } \\
\text { Himalayas in India }\end{array}$ \\
\hline Lepidium apetalum Willd. ${ }^{1}$ & Rhizomatous Ph & BP & $2120-2130$ & PU/EES/KH-15134 & $\begin{array}{l}\text { E. Europe to temp. Asia; } \\
\text { Himalayas in India }\end{array}$ \\
\hline Turritis glabra L. $^{1^{*}}$ & Ah or $\mathrm{Bh}$ & $\mathrm{BP} / \mathrm{MC}$ & $2300-2650$ & PU/EES/KH-15022 & $\begin{array}{l}\text { Temp. N. Hemisphere; W. } \\
\text { Himalayas in India }\end{array}$ \\
\hline \multicolumn{6}{|l|}{ Campanulaceae } \\
\hline Campanula cashmeriana Royle $^{1}$ & $\mathrm{Ph}$ & SA & $3150-3200$ & PU/EES/KH-15050 & Afg. to W. Himalayas to Nepal \\
\hline Campanula latifolia L. $^{1}$ & $\mathrm{Ph}$ & $\mathrm{MC} / \mathrm{SA}$ & $2525-2920$ & PU/EES/KH-15051 & $\begin{array}{l}\text { SW Siberia, W. Asia to C. } \\
\text { Himalayas }\end{array}$ \\
\hline Codonopsis ovata Benth. ${ }^{1}$ & $\mathrm{Ph}$ & $\mathrm{MC} / \mathrm{SA}$ & $2720-3800$ & PU/EES/KH-15076 & $\begin{array}{l}\text { C. Asia, Himalayas from Pak. } \\
\text { to Kashmir }\end{array}$ \\
\hline Codonopsis rotundifolia Benth. ${ }^{1}$ & Twining Ph & $\mathrm{BP}$ & $2200-2340$ & PU/EES/KH-15077 & Pak. to Himalayas \& S. Tibet \\
\hline \multicolumn{6}{|l|}{ Cannabaceae } \\
\hline Cannabis sativa L. $^{1^{*}}$ & Ah & $\mathrm{BP} / \mathrm{MC}$ & $1920-2650$ & PU/EES/KH-15052 & $\begin{array}{l}\text { Native to } C \text {. Asia now } \\
\text { cosmopolitan }\end{array}$ \\
\hline \multicolumn{6}{|l|}{ Caprifoliaceae } \\
\hline Dipsacus inermis Wall. ${ }^{1}$ & $\mathrm{Ph}$ & $\mathrm{MC} / \mathrm{SA}$ & $2700-3810$ & $\mathrm{PU} / \mathrm{EES} / \mathrm{KH}-15092$ & $\begin{array}{l}\text { Himalayas from Afg. to SW } \\
\text { China \& Mya. }\end{array}$ \\
\hline Lonicera quinquelocularis Hard. ${ }^{1}$ & ES & MC & $2500-2700$ & PU/EES/KH-1209 & E. Afg. to Himalayas \\
\hline Morina longifolia Wall. ${ }^{1}$ & $\mathrm{Ph}$ & $\mathrm{MC} / \mathrm{SA}$ & $2700-2920$ & PU/EES/KH-15147 & $\begin{array}{l}\text { N. Pakistan to Himalaya \& } \\
\text { S. Tibet }\end{array}$ \\
\hline
\end{tabular}




\begin{tabular}{|c|c|c|c|c|c|}
\hline Family/Taxon & Life-form & Forest type & OER & Voucher no. & Phytogeographic distribution \\
\hline Scabiosa speciosa Royle $^{1}$ & Ah & MC & $2720-2730$ & PU/EES/KH-15208 & $\begin{array}{l}\text { Himalayas from Pak. to } \\
\text { Uttarakhand }\end{array}$ \\
\hline Valeriana hardwickii Wall. ${ }^{1}$ & Dioecious $\mathrm{Ph}$ & $\mathrm{MC} / \mathrm{SA}$ & $2570-3140$ & PU/EES/KH-15238 & $\begin{array}{l}\text { N. Pak. to S. China \& W. } \\
\text { Malesia, Himalayas in India }\end{array}$ \\
\hline Valeriana jatamansi Jones $^{1}$ & $\mathrm{Ph}$ & $\mathrm{MC} / \mathrm{SA}$ & $2700-3150$ & PU/EES/KH-15239 & $\begin{array}{l}\text { Himalayas from Afg. to SW } \\
\text { China }\end{array}$ \\
\hline \multicolumn{6}{|l|}{ Caryophyllaceae } \\
\hline $\begin{array}{l}\text { Arenaria orbiculata Royle ex Edgew. \& } \\
\text { Hook.f. }^{1}\end{array}$ & $\mathrm{Ah}$ & MC & $2500-2600$ & PU/EES/KH-15026 & $\begin{array}{l}\text { Afg. to China; Himalayas in } \\
\text { India }\end{array}$ \\
\hline Cerastium cerastoides (L.) Britton ${ }^{1}$ & $\mathrm{Ph}$ & $\mathrm{BP} / \mathrm{MC} / \mathrm{SA}$ & $1920-3160$ & PU/EES/KH-15060 & $\begin{array}{l}\text { Temp. Eurasia, E. Canada to } \\
\text { Greenland; W. Himalayas in } \\
\text { India }\end{array}$ \\
\hline Cerastium dahuricum Fisch. ${ }^{1}$ & Scrambling Ph & $\mathrm{BP} / \mathrm{MC} / \mathrm{SA}$ & $2520-3000$ & PU/EES/KH-15061 & $\begin{array}{l}\text { European Russia to Mongolia } \\
\& \text { W. Himalayas }\end{array}$ \\
\hline Cucubalus baccifer L. ${ }^{1}$ & $\mathrm{Ph}$ & $\mathrm{BP} / \mathrm{MC} / \mathrm{SA}$ & $2400-2950$ & PU/EES/KH-15082 & Temp. Eurasia \& Himalayas \\
\hline $\begin{array}{l}\text { Lepyrodiclis holosteoides (C.A. Mey.) Fenzl } \\
\text { ex Fisch. \& C.A. Mey. }{ }^{1}\end{array}$ & Ah or Bh & $\mathrm{MC} / \mathrm{SA}$ & $2630-3120$ & PU/EES/KH-15135 & $\begin{array}{l}\text { Turkey to Mongolia \& } \\
\text { Himalayas }\end{array}$ \\
\hline Lychnis coronaria Desr. ${ }^{1^{*}}$ & $\mathrm{Ph}$ & $\mathrm{BP} / \mathrm{MC}$ & $2070-2780$ & PU/EES/KH-15141 & $\begin{array}{l}\text { EC \& SE Europe to N. Iran \& C. } \\
\text { Asia to W. Himalayas }\end{array}$ \\
\hline Silene himalayensis (Rohrb.) Majumdar ${ }^{1}$ & $\mathrm{Ah}$ & SA & $2810-2820$ & PU/EES/KH-15218 & $\begin{array}{l}\text { NE Afg. to C. China; Himalayas } \\
\text { in India }\end{array}$ \\
\hline Silene vulgaris (Moench) Garcke ${ }^{2}$ & $\mathrm{Ph}$ & $\mathrm{BP} / \mathrm{MC} / \mathrm{SA}$ & $2200-2920$ & PU/EES/KH-15219 & $\begin{array}{l}\text { Palearctic; W. Himalayas in } \\
\text { India }\end{array}$ \\
\hline Spergularia diandra (Guss.) Heldr. ${ }^{1}$ & $\mathrm{Ph}$ & MC & $2700-2710$ & PU/EES/KH-15221 & $\begin{array}{l}\text { Canary Islands, Medit. to } \\
\text { SW Siberia \& N. China; W. } \\
\text { Himalayas in India }\end{array}$ \\
\hline Stellaria decumbens Edgew. ${ }^{1}$ & $\mathrm{Ph}$ & $\mathrm{BP} / \mathrm{MC} / \mathrm{SA}$ & $2200-3150$ & PU/EES/KH-15225 & $\begin{array}{l}\text { E. \& NE Afg. to China; } \\
\text { Himalayas in India }\end{array}$ \\
\hline Stellaria media (L.) Vill. ${ }^{1^{*}}$ & $\begin{array}{l}\text { Densely or laxly } \\
\text { caespitose Ph }\end{array}$ & $\mathrm{BP} / \mathrm{MC}$ & $2250-2780$ & PU/EES/KH-15226 & $\begin{array}{l}\text { Temp. Eurasia, N. \& NE } \\
\text { Tropical Africa; throughout } \\
\text { India }\end{array}$ \\
\hline \multicolumn{6}{|l|}{ Compositae } \\
\hline Achillea millefolium $\mathrm{L}^{.^{*}}$ & Rhizomatous Ph & $\mathrm{BP} / \mathrm{MC} / \mathrm{SA}$ & $2200-3800$ & PU/EES/KH-15002 & $\begin{array}{l}\text { Subarctic \& temp. N. } \\
\text { Hemisphere to Guatemala; W. } \\
\text { Himalayas in India }\end{array}$ \\
\hline Anaphalis contorta (D.Don) Hook.f. ${ }^{1}$ & $\begin{array}{l}\text { Rhizomatous } \\
\text { under-S }\end{array}$ & $\mathrm{BP} / \mathrm{MC} / \mathrm{SA}$ & $1900-3300$ & PU/EES/KH-15014 & $\begin{array}{l}\text { Himalayas from Afg. to SW } \\
\text { China \& Mya. }\end{array}$ \\
\hline Anaphalis staintonii Georgiadou ${ }^{1}$ & $\mathrm{Ph}$ & MC & $2700-2800$ & PU/EES/KH-15015 & N. Pak. to W. Himalayas \\
\hline Anaphalis virgata Thomson ${ }^{1}$ & $\mathrm{Ph}$ & $\mathrm{BP} / \mathrm{MC} / \mathrm{SA}$ & $2200-3200$ & PU/EES/KH-15016 & Afg. to Xinjiang \& Himalayas \\
\hline Arctium lappa L. $^{1^{*}}$ & $\mathrm{Bh}$ & $\mathrm{BP} / \mathrm{SA}$ & $2300-2950$ & PU/EES/KH-15025 & $\begin{array}{l}\text { Temp. Eurasia; Himalayas } \\
\text { in India }\end{array}$ \\
\hline Artemisia absinthium $\mathrm{L}^{1^{*}}$ & $\mathrm{Ph}$ & $\mathrm{MC} / \mathrm{SA}$ & $2750-2920$ & PU/EES/KH-15030 & $\begin{array}{l}\text { Europe to Siberia \& W. } \\
\text { Himalayas }\end{array}$ \\
\hline Artemisia brevifolia Wall. ex DC. ${ }^{1}$ & SS & MC & $2450-2720$ & PU/EES/KH-15031 & $\begin{array}{l}\text { Afg. to W. Tibet \& W. } \\
\text { Himalayas }\end{array}$ \\
\hline Artemisia dubia Wall. ${ }^{1}$ & SS & $\mathrm{BP}$ & $2300-2400$ & PU/EES/KH-15032 & $\begin{array}{l}\text { Himalayas from Pak. to C. } \\
\text { Nepal \& China }\end{array}$ \\
\hline Artemisia scoparia Waldst. \& Kitam. ${ }^{1}$ & $\mathrm{Bh}$ or $\mathrm{Ph}$ & $\mathrm{MC} / \mathrm{SA}$ & $2450-3300$ & PU/EES/KH-15033 & $\begin{array}{l}\text { Palearctic region; throughout } \\
\text { India }\end{array}$ \\
\hline Artemisia vestita Wall. ex Besser ${ }^{1^{*}}$ & SS & SA & $3200-3400$ & PU/EES/KH-15034 & $\begin{array}{l}\text { Pak. to Mongolia \& China, W. } \\
\text { Himalayas in India }\end{array}$ \\
\hline Artemisia vulgaris $\mathrm{L} .^{1}$ & $\mathrm{Ph}$ & SA & $2830-2916$ & PU/EES/KH-15035 & $\begin{array}{l}\text { Temp. Eurasia to Indo-China } \\
\& \text { N. Africa }\end{array}$ \\
\hline Carduus edelbergii Rech.f. ${ }^{1^{*}}$ & $\mathrm{Ph}$ & $\mathrm{BP} / \mathrm{MC}$ & $2010-2550$ & PU/EES/KH-15056 & Afg. to Nepal \\
\hline Carpesium abrotanoides L..$^{*}$ & $\mathrm{Ph}$ & $\mathrm{BP} / \mathrm{MC}$ & $2200-2570$ & PU/EES/KH-15057 & $\begin{array}{l}\text { S. \& C. Europe to Japan \& } \\
\text { Himalayas }\end{array}$ \\
\hline Carpesium cernuum L. ${ }^{1}$ & Ah & $\mathrm{MC} / \mathrm{SA}$ & $2750-2930$ & PU/EES/KH-15055 & Eurasia; W. Himalayas in India \\
\hline Centaurea iberica Trevir..$^{*^{*}}$ & $\mathrm{Ph}$ & $\mathrm{BP}$ & $2250-2300$ & PU/EES/KH-15059 & $\begin{array}{l}\text { SE \& E. Europe to Xinjiang \& } \\
\text { W. Himalayas }\end{array}$ \\
\hline Cichorium intybus L.. ${ }^{*}$ & $\mathrm{Ph}$ & $\mathrm{BP} / \mathrm{MC}$ & $2050-2490$ & PU/EES/KH-15068 & $\begin{array}{l}\text { N. Africa, C \& SW Asia \& } \\
\text { Europe }\end{array}$ \\
\hline Cirsium arvense (L.) Scop..$^{1^{*}}$ & Dioecious Ph & $\mathrm{BP}$ & $2200-2210$ & PU/EES/KH-15070 & $\begin{array}{l}\text { Temp. Eurasia, NW Africa; } \\
\text { Himalayas in India }\end{array}$ \\
\hline Cirsium falconeri (Hook.f.) Petr. ${ }^{1}$ & $\mathrm{Ph}$ & SA & $2840-2990$ & PU/EES/KH-15071 & N. Pak. to S. Tibet \& N. Mya. \\
\hline
\end{tabular}




\begin{tabular}{|c|c|c|c|c|c|}
\hline Family/Taxon & Life-form & Forest type & OER & Voucher no. & Phytogeographic distribution \\
\hline Cirsium vulgare (Savi) Ten. ${ }^{1}$ & $\mathrm{Bh}$ & SA & $2940-2950$ & PU/EES/KH-15072 & $\begin{array}{l}\text { Europe to Siberia \& Arabian } \\
\text { Peninsula; W. Himalayas in } \\
\text { India }\end{array}$ \\
\hline Cirsium wallichii DC..$^{*}$ & $\mathrm{Ph}$ & $\mathrm{BP} / \mathrm{MC} / \mathrm{SA}$ & $1920-3210$ & PU/EES/KH-15073 & Afg. to Indian Subcontinent \\
\hline Conyza canadensis (L.) Cronquist ${ }^{1^{*}}$ & $\mathrm{Ah}$ & BP & $2010-2210$ & PU/EES/KH-15079 & $\begin{array}{l}\text { Native to Neotropic \& } \\
\text { Nearctic regions }\end{array}$ \\
\hline Crepis sancta (L.) Bornm. ${ }^{* *}$ & $\mathrm{Ah}$ & SA & $2910-2920$ & PU/EES/KH-15081 & $\begin{array}{l}\text { E. Europe, W. Asia eastwards } \\
\text { in Himalayas up to Nepal }\end{array}$ \\
\hline Doronicum roylei DC. ${ }^{1}$ & $\mathrm{Ph}$ & SA & $3800-3810$ & PU/EES/KH-15093 & $\begin{array}{l}\text { NE Pak. to Himalayas \& S. } \\
\text { Tibet }\end{array}$ \\
\hline $\begin{array}{l}\text { Erigeron multiradiatus (Lindl. ex DC.) Benth. } \\
\text { ex C. B. Clarke }{ }^{1}\end{array}$ & Rhizomatous Ph & $\mathrm{MC} / \mathrm{SA}$ & $2530-3800$ & PU/EES/KH-15101 & Afg. to China \\
\hline Lactuca macrorhiza (Royle) Hook. . . ${ }^{1}$ & Rhizomatous Ph & $\mathrm{MC} / \mathrm{SA}$ & 2570-3130 & PU/EES/KH-15125 & Afg. to Himalayas \\
\hline Lactuca dolichophylla Kitam. ${ }^{1}$ & $\mathrm{Ph}$ & MC & $2530-2540$ & PU/EES/KH-15126 & $\begin{array}{l}\text { Himalayas from Afg. to SW } \\
\text { China }\end{array}$ \\
\hline Lapsana communis L. ${ }^{1}$ & $\mathrm{Ah}$ & $\mathrm{BP} / \mathrm{MC}$ & $2315-2710$ & PU/EES/KH-15129 & $\begin{array}{l}\text { Europe to Siberia \& Iran; W. } \\
\text { Himalayas in India }\end{array}$ \\
\hline Ligularia amplexicaulis DC. ${ }^{1}$ & $\mathrm{Ph}$ & $\mathrm{MC} / \mathrm{SA}$ & $2790-2930$ & PU/EES/KH-15137 & Himalayas to S. Tibet \\
\hline Ligularia fischeri (Ledeb.) Turcz. ${ }^{1}$ & $\mathrm{Ph}$ & $\mathrm{MC} / \mathrm{SA}$ & $2570-3540$ & PU/EES/KH-15138 & $\begin{array}{l}\text { NE Pak. to S. Siberia \& Japan; } \\
\text { Himalayas in India }\end{array}$ \\
\hline Myriactis nepalensis Less. ${ }^{1}$ & $\mathrm{Ph}$ & $\mathrm{BP} / \mathrm{MC} / \mathrm{SA}$ & $1980-3000$ & $\mathrm{PU} / \mathrm{EES} / \mathrm{KH}-15150$ & $\begin{array}{l}\text { Himalayas from Afg. to SW } \\
\text { China, \& SE Asia }\end{array}$ \\
\hline Picris hieracioides Sibth. \& Sm. ${ }^{1}$ & $\mathrm{Ph}$ & $\mathrm{MC} / \mathrm{SA}$ & $2430-3000$ & PU/EES/KH-15169 & $\begin{array}{l}\text { Temp. Eurasia; Himalayas } \\
\text { in India }\end{array}$ \\
\hline Saussurea albescens Hook. f \& Thomson ${ }^{1}$ & $\mathrm{Ph}$ & SA & $3010-3020$ & PU/EES/KH-15203 & NE Afg. to Nepal \\
\hline Saussurea costus (Falc.) Lipsch. ${ }^{3}$ & $\mathrm{Ph}$ & SA & $3050-3060$ & PU/EES/KH-15206 & W. Himalayas \\
\hline Saussurea roylei C.B. Clarke ${ }^{1}$ & $\mathrm{Ph}$ & SA & $3130-3140$ & PU/EES/KH-15204 & NW Himalayas \\
\hline Saussurea taraxacifolia (Lindl.) Wall. ex DC. ${ }^{1}$ & $\mathrm{Ph}$ & SA & $3800-3810$ & PU/EES/KH-15205 & $\begin{array}{l}\text { Himalayas from Kashmir to } \\
\text { Bhutan, Xizang }\end{array}$ \\
\hline Senecio chrysanthemoides DC. ${ }^{1}$ & $\mathrm{Ph}$ & $\mathrm{MC} / \mathrm{SA}$ & $2420-3150$ & PU/EES/KH-15212 & Afg. to SC China \& Indo-China \\
\hline Serratula pallida $\mathrm{DC} .^{1}$ & $\mathrm{Ph}$ & MC & $2430-2440$ & PU/EES/KH-15213 & N. Pak. to Nepal \\
\hline Sigesbeckia orientalis L. ${ }^{1^{*}}$ & Tufted Ph & $\mathrm{BP}$ & $2200-2210$ & PU/EES/KH-15217 & E. Europe to Asia \& Australia \\
\hline Solidago virga-aurea L. $^{1}$ & $\mathrm{Ph}$ & $\mathrm{MC} / \mathrm{SA}$ & 2670-3810 & PU/EES/KH-15220 & $\begin{array}{l}\text { W. Europe to C. Siberia \& } \\
\text { Phip.; Himalayas in India }\end{array}$ \\
\hline Tanacetum multicaule Sch.Bip. ${ }^{1}$ & $\mathrm{Ph}$ & SA & $3010-3810$ & PU/EES/KH-15229 & Kashmir to SW China \\
\hline $\begin{array}{l}\text { Taraxacum officinale (L.) Weber ex } \\
\text { F.H.Wigg. }{ }^{*}\end{array}$ & $\begin{array}{c}\text { Semi-prostrate } \\
\mathrm{Ph}\end{array}$ & $\mathrm{BP} / \mathrm{MC} / \mathrm{SA}$ & $1920-3410$ & PU/EES/KH-15230 & Cosmopolitan \\
\hline Tussilago farfara $\mathrm{L}^{1}$ & $\mathrm{Ph}$ & $\mathrm{MC} / \mathrm{SA}$ & $2670-3130$ & PU/EES/KH-15236 & $\begin{array}{l}\text { Palearctic region; Himalayas } \\
\text { in India }\end{array}$ \\
\hline Xanthium spinosum L. $^{1^{*}}$ & Rhizomatous Ph & BP & $2230-2240$ & PU/EES/KH-15128 & $\begin{array}{l}\text { C. \& E. Canada to Mexico, } \\
\text { Peru to S. South America }\end{array}$ \\
\hline \multicolumn{6}{|l|}{ Convolvulaceae } \\
\hline Convolvulus arvensis L. ${ }^{1^{*}}$ & $\begin{array}{c}\text { Climbing \& } \\
\text { prostrate Ah } \\
\text { or } \mathrm{Ph}\end{array}$ & MC & $2440-2460$ & PU/EES/KH-15078 & Eurasia; throughout India \\
\hline \multicolumn{6}{|l|}{ Crassulaceae } \\
\hline Sedum ewersii Ledeb..$^{1^{*}}$ & $\mathrm{Ph}$ & SA & $3790-3810$ & PU/EES/KH-15210 & $\begin{array}{l}\text { Siberia to Afg. \& N. China; W. } \\
\text { Himalayas in India }\end{array}$ \\
\hline \multicolumn{6}{|l|}{ Cupressaceae } \\
\hline Juniperus semiglobosa Regel $^{2}$ & Monoecious ET & MC & $2450-2500$ & $\mathrm{PU} / \mathrm{EES} / \mathrm{KH}-1008$ & $\begin{array}{l}\text { SE Iran to C. Asia, Himalayas } \\
\text { from Pak. to Uttarakhand }\end{array}$ \\
\hline Juniperus squamata Buch.-Ham. ex D.Don² & $\begin{array}{l}\text { Monoecious } \\
\text { bushy, semi- } \\
\text { prostrate S/ET }\end{array}$ & SA & $3150-3440$ & $\mathrm{PU} / \mathrm{EES} / \mathrm{KH}-1015$ & N. Afg. to China \\
\hline \multicolumn{6}{|l|}{ Cyperaceae } \\
\hline Carex stenophylla Wahlenb. ${ }^{2}$ & $\begin{array}{l}\text { Rhizomatous } \\
\text { creeping Ph }\end{array}$ & SA & $2800-2920$ & PU/EES/KH-15058 & $\begin{array}{l}\text { From Caucasus \& Iran to Pak., } \\
\text { Kashmir \& Mongolia }\end{array}$ \\
\hline \multicolumn{6}{|l|}{ Dioscoreaceae } \\
\hline Dioscorea deltoidea Wall. ex Griseb. ${ }^{1}$ & Climbing Ph & $\mathrm{BP} / \mathrm{SA}$ & $1880-2810$ & PU/EES/KH-15091 & $\begin{array}{l}\text { Himalayas to SC China \& } \\
\text { Indo-China }\end{array}$ \\
\hline
\end{tabular}




\begin{tabular}{|c|c|c|c|c|c|}
\hline Family/Taxon & Life-form & Forest type & OER & Voucher no. & Phytogeographic distribution \\
\hline \multicolumn{6}{|l|}{ Elaeagnaceae } \\
\hline Hippophae rhamnoides $\mathrm{L}^{1}$ & Dioecious DT & $\mathrm{MC}$ & $2400-2500$ & $\mathrm{PU} / \mathrm{EES} / \mathrm{KH}-1204$ & $\begin{array}{l}\text { Palearctic region; W. } \\
\text { Himalayas in India }\end{array}$ \\
\hline \multicolumn{6}{|l|}{ Equisetaceae } \\
\hline Equisetum arvense $\mathrm{L}^{2}$ & $\begin{array}{l}\text { Erect or prostrate } \\
\text { rhizomatous } \mathrm{Ph}\end{array}$ & $\mathrm{BP} / \mathrm{SA}$ & $2320-3060$ & PU/EES/KH-15100 & $\begin{array}{l}\text { Subarctic \& temp. N. } \\
\text { Hemisphere }\end{array}$ \\
\hline \multicolumn{6}{|l|}{ Euphorbiaceae } \\
\hline Euphorbia esula L. ${ }^{1}$ & Erect Ph & $\mathrm{MC}$ & $2600-2760$ & PU/EES/KH-15104 & $\begin{array}{l}\text { Palearctic; W. Himalayas in } \\
\text { India }\end{array}$ \\
\hline Euphorbia pilosa $\mathrm{L} .^{1}$ & $\mathrm{Ph}$ & SA & $2920-2930$ & PU/EES/KH-15105 & C. Asia, N. Pak. to Himalayas \\
\hline Euphorbia wallichii Hook.f. ${ }^{1}$ & $\mathrm{Ph}$ & SA & $3140-3540$ & PU/EES/KH-15106 & $\begin{array}{l}\text { Himalayas from Afg. to W. } \\
\text { Himalayas to Sikkim }\end{array}$ \\
\hline \multicolumn{6}{|l|}{ Gentianaceae } \\
\hline Gentiana carinata (D.Don) Griseb. ${ }^{1}$ & $\mathrm{Ph}$ & $\mathrm{MC} / \mathrm{SA}$ & $2570-3000$ & PU/EES/KH-15111 & $\begin{array}{l}\text { Himalayas from Pak. to } \\
\text { Uttarakhand }\end{array}$ \\
\hline Gentiana moorcroftiana Wall. ex G.Don ${ }^{1}$ & $\begin{array}{l}\text { Aromatic, dwarf, } \\
\text { creeping mat } \\
\text { forming herb }\end{array}$ & SA & $3790-3800$ & PU/EES/KH-15251 & $\begin{array}{l}\text { Himalayas from Kashmir to } \\
\text { Nepal }\end{array}$ \\
\hline Gentiana tianschanica Rupr. ex Kusn. ${ }^{1}$ & $\mathrm{Ah}$ & SA & $3790-3800$ & PU/EES/KH-15112 & Himalayas \& China \\
\hline $\begin{array}{l}\text { Lomatogonium caeruleum (Royle) Harry } \\
\text { Sm. ex B.L. Burtt }\end{array}$ & Tufted Ph & SA & $3790-3810$ & PU/EES/KH-15140 & $\begin{array}{l}\text { Himalayas from Kashmir to } \\
\text { Nepal }\end{array}$ \\
\hline Swertia speciosa D.Don ${ }^{1}$ & $\mathrm{Ah}$ & SA & $2810-2820$ & PU/EES/KH-15146 & $\begin{array}{l}\text { Himalayas from Pak. to } \\
\text { Bhutan }\end{array}$ \\
\hline Swertia petiolata D. Don ${ }^{1}$ & Rhizomatous Ph & $\mathrm{BP} / \mathrm{MC} / \mathrm{SA}$ & $2310-3210$ & PU/EES/KH-15228 & E. Afg. to W. \& C. Himalayas \\
\hline \multicolumn{6}{|l|}{ Geraniaceae } \\
\hline Geranium pusillum L. ${ }^{1}$ & $\mathrm{Ph}$ & $\mathrm{BP} / \mathrm{MC} / \mathrm{SA}$ & $1920-2920$ & PU/EES/KH-15113 & Europe to W. Himalayas \\
\hline Geranium wallichianum D.Don ex Sweet ${ }^{2}$ & Ah & $\mathrm{BP} / \mathrm{MC} / \mathrm{SA}$ & $1920-3810$ & PU/EES/KH-15114 & E. Afg. to Himalayas \& Tibet \\
\hline \multicolumn{6}{|l|}{ Hamamelidaceae } \\
\hline $\begin{array}{l}\text { Parrotiopsis jacquemontiana (Decne.) } \\
\text { Rehder }^{1}\end{array}$ & DS/small DT & $\mathrm{BP}$ & $2100-2300$ & $\mathrm{PU} / \mathrm{EES} / \mathrm{KH}-1201$ & E. Afg. to W. Himalayas \\
\hline \multicolumn{6}{|l|}{ Hypericaceae } \\
\hline Hypericum perforatum $\mathrm{L} .^{.^{*}}$ & Ah or $\mathrm{Bh}$ & $\mathrm{BP} / \mathrm{MC} / \mathrm{SA}$ & $1980-3540$ & PU/EES/KH-15121 & $\begin{array}{l}\text { Europe to China, NW Africa, } \\
\text { SW Sudan; W. Himalayas in } \\
\text { India }\end{array}$ \\
\hline \multicolumn{6}{|l|}{ Iridaceae } \\
\hline Iris hookeriana Foster ${ }^{1}$ & Ah & $\mathrm{MC} / \mathrm{SA}$ & $2560-3810$ & $\mathrm{PU} / \mathrm{EES} / \mathrm{KH}-15123$ & Afg. to W. Himalayas \\
\hline \multicolumn{6}{|l|}{ Juglandaceae } \\
\hline Juglans regia $\mathrm{L}^{2}$ & DT & $\mathrm{BP}$ & $2000-2390$ & $\mathrm{PU} / \mathrm{EES} / \mathrm{KH}-1007$ & $\begin{array}{l}\text { West Asia, W. China \& } \\
\text { Himalayas }\end{array}$ \\
\hline \multicolumn{6}{|l|}{ Lamiaceae } \\
\hline Clinopodium umbrosum (M.Bieb.) Kuntze ${ }^{1^{*}}$ & $\mathrm{Ph}$ & $\mathrm{BP} / \mathrm{MC} / \mathrm{SA}$ & $2200-3000$ & PU/EES/KH-15074 & $\begin{array}{l}\text { Caucasus to N. Mya.; W. } \\
\text { Himalayas in India }\end{array}$ \\
\hline Clinopodium vulgare L.. ${ }^{*}$ & $\mathrm{Ph}$ & $\mathrm{BP} / \mathrm{MC} / \mathrm{SA}$ & $1920-3280$ & PU/EES/KH-15075 & $\begin{array}{l}\text { Medit., Europe to Siberia \& W. } \\
\text { Himalayas }\end{array}$ \\
\hline Lamium album $\mathrm{L}^{1}$ & $\mathrm{Ph}$ & $\mathrm{MC} / \mathrm{SA}$ & $2560-2930$ & PU/EES/KH-15127 & $\begin{array}{l}\text { Palearctic region; W. } \\
\text { Himalayas in India }\end{array}$ \\
\hline Nepeta erecta (Royle ex Benth.) Benth. ${ }^{1}$ & $\mathrm{Ph}$ & MC & $2700-2770$ & PU/EES/KH-15151 & E. Afg. to W. Himalayas \\
\hline Nepeta laevigata (D.Don) Hand.-Mazz. ${ }^{1}$ & $\mathrm{Ph}$ & $\mathrm{BP} / \mathrm{MC}$ & $2200-2410$ & PU/EES/KH-15152 & $\begin{array}{l}\text { Himalayas from Afg. to SW } \\
\text { China }\end{array}$ \\
\hline Nepeta linearis Royle ex Benth. ${ }^{1}$ & $\mathrm{Ph}$ & $\mathrm{MC} / \mathrm{SA}$ & $2720-3810$ & PU/EES/KH-15153 & E. Afg. to W. Himalayas \\
\hline Origanum vulgare L.. ${ }^{*}$ & $\mathrm{Ph}$ & $\mathrm{BP} / \mathrm{MC} / \mathrm{SA}$ & $2310-3210$ & PU/EES/KH-15155 & Eurasia; Himalayas in India \\
\hline Phlomis bracteosa Royle ex Benth. ${ }^{1}$ & Rhizomatous Ph & SA & $2920-3800$ & PU/EES/KH-15166 & E. Afg. to Himalayas \\
\hline Phlomis cashmeriana Royle ex Benth. ${ }^{1}$ & $\mathrm{Ph}$ & $\mathrm{BP} / \mathrm{MC} / \mathrm{SA}$ & $2310-2910$ & PU/EES/KH-15167 & Afg. to W. Himalayas \\
\hline Prunella vulgaris $\mathrm{L.}^{.^{*}}$ & $\mathrm{Ph}$ & $\mathrm{BP} / \mathrm{MC} / \mathrm{SA}$ & $1920-3150$ & PU/EES/KH-15191 & $\begin{array}{l}\text { Europe, N. Africa, N. America } \\
\& \text { Asia }\end{array}$ \\
\hline Salvia hians Royle ex Benth. ${ }^{1}$ & Erect Ph & MC & $2590-2600$ & PU/EES/KH-15198 & $\begin{array}{l}\text { Himalayas from Kashmir to } \\
\text { Nepal }\end{array}$ \\
\hline
\end{tabular}




\begin{tabular}{|c|c|c|c|c|c|}
\hline Family/Taxon & Life-form & Forest type & OER & Voucher no. & Phytogeographic distribution \\
\hline Salvia moorcroftiana Wall. ex Benth. ${ }^{1}$ & Aromatic Ph & $\mathrm{MC}$ & $2720-2730$ & PU/EES/KH-15199 & $\begin{array}{l}\text { Himalayas from Pak. to W. } \\
\text { Nepal }\end{array}$ \\
\hline Salvia nubicola Wall. ex Sweet ${ }^{1}$ & $\mathrm{Ph}$ & $\mathrm{MC} / \mathrm{SA}$ & $2700-2920$ & PU/EES/KH-15200 & E. Afg. to Himalayas \\
\hline Stachys floccosa Benth. ${ }^{1}$ & Erect $\mathrm{Ph}$ & $\mathrm{BP} / \mathrm{MC} / \mathrm{SA}$ & $2390-2710$ & PU/EES/KH-15223 & $\begin{array}{l}\text { Himalayas from Afg., Pak. to } \\
\text { Kashmir }\end{array}$ \\
\hline Stachys sericea Wall. ex Benth. ${ }^{1}$ & $\mathrm{Ph}$ & SA & $2920-2930$ & PU/EES/KH-15224 & Kashmir to SE Tibet \\
\hline Thymus linearis Benth..$^{{ }^{*}}$ & Ah & $\mathrm{MC} / \mathrm{SA}$ & $2500-3000$ & PU/EES/KH-15250 & $\begin{array}{l}\text { N. Iran to Xinjiang \& } \\
\text { Himalayas }\end{array}$ \\
\hline \multicolumn{6}{|l|}{ Leguminosae } \\
\hline $\begin{array}{l}\text { Argyrolobium flaccidum (Royle) Jaub. \& } \\
\text { Spach }^{1}\end{array}$ & Prostrate Ph & $\mathrm{MC}$ & $2400-2550$ & PU/EES/KH-15027 & India, Nepal \& Pak. \\
\hline Lathyrus humilis (Ser.) Spreng. ${ }^{1}$ & Ah or $\mathrm{Ph}$ & SA & $3110-3120$ & PU/EES/KH-15130 & $\begin{array}{l}\text { E. Europe to temp. Asia \& W. } \\
\text { Himalayas }\end{array}$ \\
\hline Lathyrus laevigatus (Waldst. \& Kit.) Gren. ${ }^{1}$ & $\mathrm{Ph}$ & $\mathrm{MC} / \mathrm{SA}$ & $2670-3060$ & PU/EES/KH-15131 & $\begin{array}{l}\text { Europe, Himalayas from Pak. } \\
\text { to W. Nepal }\end{array}$ \\
\hline Lathyrus pratensis L. $^{2}$ & $\mathrm{Ph}$ & SA & $2830-2840$ & PU/EES/KH-15132 & $\begin{array}{l}\text { Europe to Mongolia \& } \\
\text { Himalayas, Morocco, Ethiopia } \\
\text { \& Yemen }\end{array}$ \\
\hline Leonurus cardiaca L. $^{1}$ & Scrambling Ph & SA & $2920-2930$ & PU/EES/KH-15133 & $\begin{array}{l}\text { Europe, Himalayas from Pak. } \\
\text { to Nepal }\end{array}$ \\
\hline Lespedeza cuneata (Dum.Cours.) G.Don² & Ah & $\mathrm{BP}$ & $1980-1990$ & PU/EES/KH-15136 & $\begin{array}{l}\text { Afg. to Japan \& tropical Asia, } \\
\text { E. \& SE Australia }\end{array}$ \\
\hline Medicago sativa Linn $^{1}{ }^{1}$ & $\begin{array}{c}\text { Prostrate or } \\
\text { decumbent } \mathrm{Ph}\end{array}$ & $\mathrm{BP}$ & $1920-1930$ & PU/EES/KH-15143 & $\begin{array}{l}\text { Europe to Mongolia \& Indian } \\
\text { Subcontinent }\end{array}$ \\
\hline Medicago lupulina L. $^{1^{*}}$ & $\begin{array}{c}\text { Erect or } \\
\text { procumbent Ph }\end{array}$ & $\mathrm{BP} / \mathrm{MC}$ & $1880-2720$ & PU/EES/KH-15144 & Asia, Africa \& Europe \\
\hline Medicago minima (L.) L. ${ }^{1}$ & Ah or $\mathrm{Ph}$ & MC & $2770-2780$ & PU/EES/KH-15145 & $\begin{array}{l}\text { Temp. Eurasia to India, } \\
\text { tropical Africa to SW. Arabian } \\
\text { Peninsula }\end{array}$ \\
\hline Oxytropis cachemiriana Cambess. ${ }^{2}$ & $\begin{array}{l}\text { Creeping annual } \\
\text { or short-lived } \mathrm{Ph}\end{array}$ & SA & $3790-3800$ & PU/EES/KH-15160 & N. Pak. to W. Himalayas \\
\hline Oxytropis mollis Benth. ${ }^{1}$ & $\mathrm{Ph}$ & SA & $3790-3800$ & PU/EES/KH-15162 & India, Pakistan \& Xizang \\
\hline Robinia pseudoacacia L. $^{2^{*}}$ & DT & MC & $2330-2340$ & PU/EES/KH-1012 & Native to N. America \\
\hline Trifolium pratense ${\mathrm{L} . \mathrm{.}^{*}}^{*}$ & $\mathrm{Ph}$ & $\mathrm{BP} / \mathrm{MC}$ & $1980-2710$ & PU/EES/KH-15234 & $\begin{array}{l}\text { Europe \& N. Asia, Himalayas } \\
\text { in India }\end{array}$ \\
\hline Trifolium repens L..1* & $\begin{array}{c}\text { Erect to } \\
\text { decumbent Ph }\end{array}$ & $\mathrm{BP} / \mathrm{MC} / \mathrm{SA}$ & $1920-3540$ & PU/EES/KH-15235 & $\begin{array}{l}\text { Macaronesia, NW Africa, } \\
\text { Egypt to Zimbabwe, Europe to } \\
\text { Mongolia \& Himalayas }\end{array}$ \\
\hline Trigonella emodi Benth. ${ }^{1}$ & $\mathrm{Ph}$ & SA & $3800-3810$ & PU/EES/KH-15232 & Afg. to Himalayas \\
\hline Vicia sativa L. $^{2^{*}}$ & $\mathrm{Bh}$ & $\mathrm{MC} / \mathrm{SA}$ & $2780-3120$ & PU/EES/KH-15243 & Kashmir to Eurasia \\
\hline \multicolumn{6}{|l|}{ Liliaceae } \\
\hline Fritillaria roylei Hook. ${ }^{1}$ & $\mathrm{Ph}$ & SA & $3800-3900$ & PU/EES/KH-15252 & Pak. to C. China \\
\hline \multicolumn{6}{|l|}{ Malvaceae } \\
\hline Malva neglecta Wallr. ${ }^{1^{*}}$ & $\mathrm{Ph}$ & $\mathrm{BP} / \mathrm{MC} / \mathrm{SA}$ & $2310-2940$ & PU/EES/KH-15142 & $\begin{array}{l}\text { Canary Islands, Morocco, } \\
\text { Europe to C. Asia \& W. } \\
\text { Himalayas }\end{array}$ \\
\hline \multicolumn{6}{|l|}{ Melanthiaceae } \\
\hline Trillium govanianum Wall. ex D.Don ${ }^{5}$ & $\begin{array}{c}\text { Erect or } \\
\text { spreading Ph }\end{array}$ & SA & $3050-3310$ & PU/EES/KH-15233 & E. Afg. to Himalayas \\
\hline \multicolumn{6}{|l|}{ Oleaceae } \\
\hline Syringa emodi Wall. ex Royle ${ }^{1}$ & DT & MC & $2450-2500$ & PU/EES/KH-1205 & Pak. to Nepal \& Tibet \\
\hline \multicolumn{6}{|l|}{ Onagraceae } \\
\hline Circaea alpina $\mathrm{L}^{1}$ & Rhizomatous Ph & $\mathrm{BP} / \mathrm{MC} / \mathrm{SA}$ & $2380-3000$ & PU/EES/KH-15069 & Temp. N. Hemisphere \\
\hline Epilobium hirsutum $\mathrm{L}^{2^{*}}$ & $\mathrm{Ph}$ & $\mathrm{BP} / \mathrm{MC} / \mathrm{SA}$ & $2200-3150$ & PU/EES/KH-15097 & $\begin{array}{l}\text { Temp. Eurasia to Africa; W. } \\
\text { Himalayas in India }\end{array}$ \\
\hline Epilobium laxum Royle $^{1}$ & $\mathrm{Ph}$ & SA & $2980-2990$ & PU/EES/KH-15098 & C. Asia to W. Himalayas \\
\hline Oenothera rosea L'Hér. ex Aiton ${ }^{1^{*}}$ & $\mathrm{Ph}$ & $\mathrm{BP} / \mathrm{MC} / \mathrm{SA}$ & $2230-2930$ & PU/EES/KH-15154 & Native to C. \& S. America \\
\hline \multicolumn{6}{|l|}{ Orchidaceae } \\
\hline Cypripedium cordigerum D.Don ${ }^{6}$ & $\mathrm{Ph}$ & SA & $2950-2960$ & PU/EES/KH-15087 & $\begin{array}{l}\text { N. Pak. to Himalayas \& S. } \\
\text { Tibet }\end{array}$ \\
\hline
\end{tabular}




\begin{tabular}{|c|c|c|c|c|c|}
\hline Family/Taxon & Life-form & Forest type & OER & Voucher no. & Phytogeographic distribution \\
\hline Epipactis helleborine (L.) Crantz ${ }^{1}$ & Rhizomatous Ph & $\mathrm{BP} / \mathrm{MC} / \mathrm{SA}$ & $2330-2960$ & PU/EES/KH-15096 & $\begin{array}{l}\text { NW Africa, Europe to China; } \\
\text { Himalayas in India }\end{array}$ \\
\hline Epipactis royleana Lindl. $^{1}$ & Rhizomatous Ph & $\mathrm{MC} / \mathrm{SA}$ & $2700-2920$ & PU/EES/KH-15099 & E. Afg. to C. Asia \& Himalayas \\
\hline \multicolumn{6}{|l|}{ Orobanchaceae } \\
\hline Orobanche alba Stephan ${ }^{1}$ & $\begin{array}{l}\text { Rhizomatous } \\
\text { aromatic } \mathrm{Ph}\end{array}$ & $\mathrm{MC} / \mathrm{SA}$ & $2770-3160$ & PU/EES/KH-15156 & $\begin{array}{l}\text { Europe, Afg., Pak., W. } \\
\text { Himalayas \& Tibet }\end{array}$ \\
\hline Pedicularis pectinata Wall. ex Benn. ${ }^{1}$ & $\mathrm{Ph}$ & $\mathrm{BP} / \mathrm{MC} / \mathrm{SA}$ & 2310-3810 & PU/EES/KH-15163 & $\begin{array}{l}\text { W. Himalayas from Pak. to } \\
\text { W. Nepal }\end{array}$ \\
\hline \multicolumn{6}{|l|}{ Oxalidaceae } \\
\hline Oxalis acetosella L. ${ }^{1}$ & Tufted Ph & $\mathrm{BP} / \mathrm{MC} / \mathrm{SA}$ & $1880-3120$ & $\mathrm{PU} / \mathrm{EES} / \mathrm{KH}-15158$ & $\begin{array}{l}\text { Europe to Japan; W. } \\
\text { Himalayas in India }\end{array}$ \\
\hline Oxalis corniculata $\mathrm{L}^{.^{*}}$ & Rhizomatous Ph & $\mathrm{BP} / \mathrm{MC} / \mathrm{SA}$ & $1880-2950$ & PU/EES/KH-15159 & Cosmopolitan \\
\hline \multicolumn{6}{|l|}{ Papaveraceae } \\
\hline Corydalis stewartii Fedde ${ }^{1}$ & $\begin{array}{l}\text { Rhizomatous Ah } \\
\text { or Bh }\end{array}$ & BP & $2200-2210$ & PU/EES/KH-15080 & Afg. to Nepal \\
\hline \multicolumn{6}{|l|}{ Phytolaccaceae } \\
\hline Phytolacca acinosa Roxb. $^{1}$ & $\mathrm{Ph}$ & $\mathrm{BP} / \mathrm{MC}$ & $2270-2500$ & PU/EES/KH-15168 & Kashmir to SW China \\
\hline \multicolumn{6}{|l|}{ Pinaceae } \\
\hline Abies pindrow (Royle ex D.Don) Royle ${ }^{2}$ & Coniferous ET & $\mathrm{BP} / \mathrm{MC} / \mathrm{SA}$ & $2220-3300$ & $\mathrm{PU} / \mathrm{EES} / \mathrm{KH}-1001$ & N. Afghanistan to Nepal \\
\hline Cedrus deodara (Roxb. ex D.Don) G.Don² & Coniferous ET & BP & $1810-2200$ & PU/EES/KH-1005 & $\begin{array}{l}\text { NE Afg. to W. Nepal \& NW } \\
\text { India }\end{array}$ \\
\hline Picea smithiana (Wall.) Boiss. ${ }^{2}$ & Coniferous ET & $\mathrm{BP} / \mathrm{MC} / \mathrm{SA}$ & $2000-2960$ & $\mathrm{PU} / \mathrm{EES} / \mathrm{KH}-1009$ & NE Afg. to C. Himalayas \\
\hline Pinus wallichiana A.B.Jacks. ${ }^{2}$ & Coniferous ET & $\mathrm{BP} / \mathrm{MC} / \mathrm{SA}$ & $1800-3140$ & $\mathrm{PU} / \mathrm{EES} / \mathrm{KH}-1010$ & Himalayas from Afg. to Tibet \\
\hline \multicolumn{6}{|l|}{ Plantaginaceae } \\
\hline Plantago lanceolata L. $^{1^{*}}$ & $\mathrm{Ph}$ & $\mathrm{BP} / \mathrm{MC} / \mathrm{SA}$ & $1920-2930$ & PU/EES/KH-15172 & $\begin{array}{l}\text { Palearctic \& Nearctic regions; } \\
\text { Himalayas in India }\end{array}$ \\
\hline Plantago major $\mathrm{L}^{2^{*}}$ & $\mathrm{Ph}$ & $\mathrm{BP} / \mathrm{MC} / \mathrm{SA}$ & $2200-3160$ & PU/EES/KH-15173 & $\begin{array}{l}\text { Europe, N. \& C. Asia, } \\
\text { introduced all over the world }\end{array}$ \\
\hline Veronica laxa Benth. ${ }^{1}$ & $\mathrm{Ph}$ & $\mathrm{BP} / \mathrm{MC} / \mathrm{SA}$ & $2120-3150$ & $\mathrm{PU} / \mathrm{EES} / \mathrm{KH}-15240$ & $\begin{array}{l}\text { N. Pak. to Nepal, C. \& S. China } \\
\text { \& Japan; W. Himalayas in India }\end{array}$ \\
\hline Veronica persica Poir. ${ }^{1^{*}}$ & $\mathrm{Ph}$ & SA & $2950-2960$ & PU/EES/KH-15241 & $\begin{array}{l}\text { Native to Iran, now a } \\
\text { worldwide weed; Himalayas } \\
\text { in India }\end{array}$ \\
\hline \multicolumn{6}{|l|}{ Poaceae } \\
\hline Agrostis gigantea Roth $^{1}$ & Rhizomatous Ph & $\mathrm{BP} / \mathrm{MC} / \mathrm{SA}$ & $2250-2850$ & $\mathrm{PU} / \mathrm{EES} / \mathrm{KH}-15010$ & $\begin{array}{l}\text { Palearctic region, introduced } \\
\text { in Nearctic; Himalayas in India }\end{array}$ \\
\hline Brachypodium sylvaticum (Huds.) P.Beauv. ${ }^{1}$ & Tufted Ph & $\mathrm{BP} / \mathrm{MC}$ & $2250-2510$ & PU/EES/KH-15040 & Eurasia; throughout India \\
\hline Bromus inermis Leyss..$^{*}$ & Rhizomatous Ph & $\mathrm{BP} / \mathrm{MC}$ & $2050-2760$ & PU/EES/KH-15041 & $\begin{array}{l}\text { Palearctic \& Nearctic regions; } \\
\text { W. Himalayas in India }\end{array}$ \\
\hline Bromus japonicus Thunb..$^{*}$ & Ah & $\mathrm{BP} / \mathrm{MC} / \mathrm{SA}$ & $2250-2950$ & PU/EES/KH-15042 & $\begin{array}{l}\text { Medit. to temp. Eurasia; W. } \\
\text { Himalayas in India }\end{array}$ \\
\hline Bromus pectinatus Thunb. $^{1}$ & $\mathrm{Ah}$ & $\mathrm{BP} / \mathrm{MC} / \mathrm{SA}$ & $2250-2300$ & $\mathrm{PU} / \mathrm{EES} / \mathrm{KH}-15043$ & $\begin{array}{l}\text { Europe, Iran \& Afg. eastwards } \\
\text { through India to China, Pak., } \\
\text { Sudan through Ethiopia to } \\
\text { Egypt, Sinai \& Arabia }\end{array}$ \\
\hline Bromus tomentosus Trin. ${ }^{1}$ & Rhizomatous Ph & $\mathrm{BP} / \mathrm{MC}$ & $2250-2800$ & PU/EES/KH-15044 & $\begin{array}{l}\text { Medit. to Xinjiang \& Pak.; W. } \\
\text { Himalayas in India }\end{array}$ \\
\hline $\begin{array}{l}\text { Calamagrostis pseudophragmites (Haller) } \\
\text { Koeler }^{2}\end{array}$ & $\begin{array}{l}\text { Creeping } \\
\text { rhizomatous } \\
\text { tufted } \mathrm{Ph} \\
\end{array}$ & $\mathrm{MC} / \mathrm{SA}$ & $2450-3800$ & PU/EES/KH-15049 & $\begin{array}{l}\text { Europe to Japan \& Himalaya; } \\
\text { Himalayas in India }\end{array}$ \\
\hline Cynodon dactylon (L.) Pers. ${ }^{1}$ & $\begin{array}{l}\text { Stoloniferous Ph } \\
\text { with rhizomes }\end{array}$ & $\mathrm{BP} / \mathrm{MC} / \mathrm{SA}$ & $1920-2930$ & PU/EES/KH-15083 & $\begin{array}{l}\text { Temp. \& Subtropical } \\
\text { Old World to Australia; } \\
\text { throughout India }\end{array}$ \\
\hline Elymus dahuricus Griseb. ${ }^{1}$ & Tufted Ph & MC & $2430-2780$ & $\mathrm{PU} / \mathrm{EES} / \mathrm{KH}-15094$ & Temp. Asia; Himalayas in India \\
\hline Koeleria macrantha (Ledeb.) Schult.. ${ }^{1^{*}}$ & Rhizomatous Ph & $\mathrm{MC} / \mathrm{SA}$ & $2460-3810$ & PU/EES/KH-15124 & $\begin{array}{l}\text { Temp. N. Hemisphere to } \\
\text { Mexico; Himalayas in India }\end{array}$ \\
\hline Lolium perenne L.. ${ }^{*}$ & $\mathrm{Ph}$ & MC & $2420-2430$ & PU/EES/KH-15139 & $\begin{array}{l}\text { N. Africa, Europe to Siberia \& } \\
\text { Himalayas }\end{array}$ \\
\hline Oryzopsis gracilis (Mez) Pilg. ${ }^{1}$ & Ah or $\mathrm{Ph}$ & $\mathrm{BP} / \mathrm{MC}$ & $1920-2630$ & PU/EES/KH-15157 & Iran to China \\
\hline Phleum alpinum $\mathrm{L}^{2}$ & $\begin{array}{l}\text { Trailing or } \\
\text { creeping Ph }\end{array}$ & $\mathrm{BP} / \mathrm{MC} / \mathrm{SA}$ & $2250-3140$ & PU/EES/KH-15165 & $\begin{array}{l}\text { Palearctic \& Nearctic regions; } \\
\text { Himalayas in India }\end{array}$ \\
\hline
\end{tabular}




\begin{tabular}{|c|c|c|c|c|c|}
\hline Family/Taxon & Life-form & Forest type & OER & Voucher no. & Phytogeographic distribution \\
\hline Poa alpina $\mathrm{L}^{1}$ & $\mathrm{Ph}$ & $\mathrm{BP} / \mathrm{MC} / \mathrm{SA}$ & $1980-3150$ & PU/EES/KH-15174 & $\begin{array}{l}\text { Temp. N. Hemisphere to } \\
\text { Mexico; W. Himalayas in India }\end{array}$ \\
\hline Poa pratensis L..2* & Tufted Ph & $\mathrm{BP} / \mathrm{MC} / \mathrm{SA}$ & $2070-2990$ & PU/EES/KH-15175 & $\begin{array}{l}\text { Palearctic \& Nearctic region; } \\
\text { Himalayas in India }\end{array}$ \\
\hline Polypogon fugax Nees ex Steud. ${ }^{1^{*}}$ & $\mathrm{Ph}$ & $\mathrm{BP} / \mathrm{MC} / \mathrm{SA}$ & $2310-3000$ & PU/EES/KH-15180 & $\begin{array}{l}\text { Iraq to Mya. mainly in } \\
\text { Himalayas \& C. Asia }\end{array}$ \\
\hline Setaria viridis (L.) P.Beauv..$^{1^{*}}$ & $\mathrm{Bh}$ or $\mathrm{Ph}$ & $\mathrm{BP}$ & $2360-2370$ & PU/EES/KH-15215 & Palearctic; Himalayas in India \\
\hline Stipa sibirica (L.) Lam. ${ }^{1}$ & $\begin{array}{l}\text { Caespitose or } \\
\text { tufted Ah }\end{array}$ & $\mathrm{BP} / \mathrm{MC}$ & $1920-2770$ & PU/EES/KH-15227 & Temp. Asia to Himalayas \\
\hline Vulpia myuros (L.) C.C.Gmel..$^{{ }^{*}}$ & Prostrate Ph & $\mathrm{BP} / \mathrm{MC}$ & $2260-2450$ & PU/EES/KH-15249 & $\begin{array}{l}\text { Europe to Taiwan \& Sri Lanka., } \\
\text { Arabian Peninsula \& Kenya; } \\
\text { throughout India }\end{array}$ \\
\hline \multicolumn{6}{|l|}{ Polemoniaceae } \\
\hline Polemonium caeruleum L. ${ }^{1}$ & Ah & $\mathrm{MC} / \mathrm{SA}$ & $2590-2960$ & PU/EES/KH-15178 & $\begin{array}{l}\text { Europe to C. Siberia \& } \\
\text { Caucasus, Himalayas from } \\
\text { Pak. to W. Nepal }\end{array}$ \\
\hline \multicolumn{6}{|l|}{ Polygonaceae } \\
\hline Aconogonon alpinum (All.) Schur ${ }^{1}$ & $\mathrm{Ph}$ & $\mathrm{BP}$ & $2300-2400$ & PU/EES/KH-15003 & $\begin{array}{l}\text { Palearctic; W. Himalayas in } \\
\text { India }\end{array}$ \\
\hline Bistorta amplexicaulis (D.Don) Greene ${ }^{1}$ & Erect $\mathrm{Ph}$ & $\mathrm{BP} / \mathrm{MC} / \mathrm{SA}$ & $2300-3000$ & PU/EES/KH-15039 & $\begin{array}{l}\text { E. Afg. to C. China; Himalayas } \\
\text { in India }\end{array}$ \\
\hline Oxyria digyna (L.) Hill ${ }^{1}$ & $\mathrm{Ph}$ & SA & $2830-3160$ & PU/EES/KH-15161 & $\begin{array}{l}\text { Palearctic \& Nearctic regions; } \\
\text { Himalayas in India }\end{array}$ \\
\hline $\begin{array}{l}\text { Persicaria capitata (Buch.-Ham. ex D.Don) } \\
\text { H.Gross }^{1}\end{array}$ & $\mathrm{Ph}$ & $\mathrm{BP} / \mathrm{MC} / \mathrm{SA}$ & $2200-3150$ & PU/EES/KH-15164 & $\begin{array}{l}\text { Indian Subcontinent to S. } \\
\text { China \& Indo-China }\end{array}$ \\
\hline Polygonum aviculare L. $^{1^{*}}$ & $\mathrm{Ph}$ & $\mathrm{BP} / \mathrm{MC} / \mathrm{SA}$ & $2210-2950$ & PU/EES/KH-15177 & $\begin{array}{l}\text { Palearctic \& Nearctic regions; } \\
\text { Himalayas in India }\end{array}$ \\
\hline Polygonum filiforme Thunb. ${ }^{1}$ & $\mathrm{Ph}$ & $\mathrm{BP}$ & 1920-1930 & PU/EES/KH-15179 & $\begin{array}{l}\text { Japan, Korea, India, Mya., } \\
\text { Phip. \& Vietnam }\end{array}$ \\
\hline Rheum webbianum Royle $^{1}$ & $\mathrm{Ph}$ & SA & $3790-3800$ & PU/EES/KH-15196 & Himalayas from Pak. to Nepal \\
\hline Rumex nepalensis Spreng..$^{*}$ & Erect $\mathrm{Ph}$ & $\mathrm{BP} / \mathrm{MC} / \mathrm{SA}$ & $1920-3410$ & PU/EES/KH-15197 & $\begin{array}{l}\text { Afg., India, Pak., Persia, SW } \\
\text { China, Turkey, N. Africa \& Italy }\end{array}$ \\
\hline \multicolumn{6}{|l|}{ Primulaceae } \\
\hline Androsace rotundifolia $\mathrm{Sm} .{ }^{1}$ & Rhizomatous Ph & MC & $2600-2750$ & PU/EES/KH-15017 & Afg., Tibet \& W. Himalayas \\
\hline Androsace sarmentosa Wall. ${ }^{1}$ & $\mathrm{Ph}$ & MC & $2700-2800$ & PU/EES/KH-15018 & $\begin{array}{l}\text { Indian Himalayas, Nepal \& } \\
\text { Tibet }\end{array}$ \\
\hline Primula macrophylla D. Don ${ }^{1}$ & Erect Ph & $\mathrm{MC} / \mathrm{SA}$ & $2720-3150$ & PU/EES/KH-15190 & $\begin{array}{l}\text { Himalayas from Afg. to SE } \\
\text { Tibet }\end{array}$ \\
\hline \multicolumn{6}{|l|}{ Pteridaceae } \\
\hline Adiantum capillus-veneris L. ${ }^{2}$ & $\begin{array}{l}\text { Epilithic } \\
\text { perennial fern }\end{array}$ & $\mathrm{BP} / \mathrm{MC} / \mathrm{SA}$ & 1950-3000 & PU/EES/KH-15007 & $\begin{array}{l}\text { Nearctic, Neotropical, } \\
\text { Afrotropical, Australasian, } \\
\text { Indomalayan \& Palearctic } \\
\text { regions; throughout India }\end{array}$ \\
\hline Pteris cretica L. ${ }^{1}$ & Rhizomatous Ph & $\mathrm{BP}$ & $2370-2380$ & PU/EES/KH-15192 & $\begin{array}{l}\text { S. Africa, Europe to E. Asia; } \\
\text { throughout India }\end{array}$ \\
\hline \multicolumn{6}{|l|}{ Ranunculaceae } \\
\hline Aconitum chasmanthum Stapf ex Holmes ${ }^{3}$ & $\mathrm{Ph}$ & SA & $3200-3800$ & PU/EES/KH-15004 & $\begin{array}{l}\text { Himalayas from Pak. to Nepal } \\
\& \text { Mongolia }\end{array}$ \\
\hline Aconitum heterophyllum Wall. ex Royle ${ }^{5}$ & Rhizomatous Ph & $\mathrm{MC} / \mathrm{SA}$ & $2700-3810$ & PU/EES/KH-15005 & $\begin{array}{l}\text { Himalayas from Pak. to C. } \\
\text { Nepal }\end{array}$ \\
\hline Actaea spicata L. $^{1}$ & Rhizomatous Ph & $\mathrm{MC} / \mathrm{SA}$ & $2500-2931$ & PU/EES/KH-15006 & E. Afg. to Himalaya \\
\hline Anemone obtusiloba Lindl. $^{1}$ & $\mathrm{Ph}$ & SA & $3200-3300$ & PU/EES/KH-15019 & $\begin{array}{l}\text { Himalayas, Mongolia, NC } \\
\text { China \& Kazakhstan }\end{array}$ \\
\hline Aquilegia pubiflora Wall. ex Royle ${ }^{1}$ & $\mathrm{Ph}$ & $\mathrm{MC} / \mathrm{SA}$ & $2500-3200$ & $\mathrm{PU} / \mathrm{EES} / \mathrm{KH}-15020$ & Afg., Pak., \& W. Himalayas \\
\hline Caltha palustris $\mathrm{L}^{2}$ & $\mathrm{Ph}$ & $\mathrm{MC} / \mathrm{SA}$ & $2800-2950$ & PU/EES/KH-15048 & $\begin{array}{l}\text { Palearctic \& Nearctic regions; } \\
\text { Himalayas in India }\end{array}$ \\
\hline Delphinium roylei Munz ${ }^{1}$ & $\mathrm{Ph}$ & $\mathrm{BP}$ & $2200-2210$ & PU/EES/KH-15088 & Pak. \& Kashmir \\
\hline Delphinium vestitum Wall. ex Royle ${ }^{1}$ & $\mathrm{Ph}$ & $\mathrm{MC} / \mathrm{SA}$ & $2520-3120$ & PU/EES/KH-15089 & $\begin{array}{l}\text { Himalayas from Pak. to } \mathrm{E} \text {. } \\
\text { Nepal }\end{array}$ \\
\hline Ranunculus hirtellus Royle ${ }^{1}$ & Rhizomatous Eh & $\mathrm{BP} / \mathrm{MC}$ & $2250-2780$ & PU/EES/KH-15193 & $\begin{array}{l}\text { Himalayas from Kashmir to } \\
\text { Sikkim, Tibet \& W. China }\end{array}$ \\
\hline $\begin{array}{l}\text { Ranunculus laetus Wall. ex Hook. f. \& J.W. } \\
\text { Thomson }^{1^{*}}\end{array}$ & $\mathrm{Ph}$ & $\mathrm{BP} / \mathrm{MC} / \mathrm{SA}$ & $2200-2990$ & PU/EES/KH-15194 & $\begin{array}{l}\text { Himalayas from Afg. to SW } \\
\text { China }\end{array}$ \\
\hline
\end{tabular}




\begin{tabular}{|c|c|c|c|c|c|}
\hline Family/Taxon & Life-form & Forest type & OER & Voucher no. & Phytogeographic distribution \\
\hline Ranunculus palmatifidus RiedI $^{1}$ & Erect $\mathrm{Ph}$ & $\mathrm{BP} / \mathrm{MC} / \mathrm{SA}$ & $2310-2930$ & PU/EES/KH-15195 & W. Himalayas \\
\hline Thalictrum minus L.. ${ }^{*}$ & $\mathrm{Ph}$ & $\mathrm{BP}$ & $2310-2340$ & PU/EES/KH-15231 & $\begin{array}{l}\text { Himalayas from Pak. to Nepal } \\
\text { \& temp. Eurasia }\end{array}$ \\
\hline \multicolumn{6}{|l|}{ Rosaceae } \\
\hline Agrimonia pilosa Ledeb. ${ }^{1}$ & Rhizomatous Ph & $\mathrm{BP} / \mathrm{MC}$ & $2200-2600$ & PU/EES/KH-15011 & $\begin{array}{l}\text { N. \& EC Europe to Japan \& N. } \\
\text { Indo-China }\end{array}$ \\
\hline Alchemilla trollii Rothm ${ }^{1}$ & $\mathrm{Ph}$ & $\mathrm{MC} / \mathrm{SA}$ & $2750-3000$ & PU/EES/KH-15012 & W. Himalayas \& Pak. \\
\hline Crataegus songarica K. Koch ${ }^{2 *}$ & DS/small DT & $\mathrm{BP}$ & $2100-2200$ & $\mathrm{PU} / \mathrm{EES} / \mathrm{KH}-1202$ & $\begin{array}{l}\text { Iran to NW China \& W. } \\
\text { Himalayas }\end{array}$ \\
\hline Filipendula vestita (Wall. ex G. Don) Maxim. ${ }^{1}$ & $\mathrm{Ph}$ & MC & $2420-2780$ & PU/EES/KH-15107 & $\begin{array}{l}\text { Afg., Pak., Nepal \& W. } \\
\text { Himalayas }\end{array}$ \\
\hline $\begin{array}{l}\text { Fragaria nubicola (Hook. f.) Lindl. ex } \\
\text { Lacaita }^{1^{*}}\end{array}$ & Stoloniferous Ph & $\mathrm{BP} / \mathrm{MC} / \mathrm{SA}$ & $1880-3540$ & PU/EES/KH-15108 & Himalayas from Afg. to Mya. \\
\hline Geum elatum Wall. ex G. Don ${ }^{1}$ & Rhizomatous Ph & $\mathrm{MC} / \mathrm{SA}$ & $2720-3800$ & PU/EES/KH-15115 & $\begin{array}{l}\text { Himalayas from Pak. to SE } \\
\text { Tibet \& SC China }\end{array}$ \\
\hline Geum roylei Wall. ex F.Bolle ${ }^{1}$ & $\mathrm{Ph}$ & $\mathrm{BP} / \mathrm{MC} / \mathrm{SA}$ & $2200-3120$ & PU/EES/KH-15116 & $\begin{array}{l}\text { Himalayas from Afg. to C. } \\
\text { Nepal }\end{array}$ \\
\hline Potentilla indica (Andrews) Th.Wolf ${ }^{1}$ & $\mathrm{Ph}$ & $\mathrm{BP} / \mathrm{MC}$ & $2120-2790$ & PU/EES/KH-15187 & $\begin{array}{l}\text { Indomalayan, E. Asia, Indian } \\
\text { Himalayas }\end{array}$ \\
\hline Potentilla anserina $\mathrm{L}^{2}$ & $\mathrm{Ph}$ & $\mathrm{MC} / \mathrm{SA}$ & $2790-3000$ & $\mathrm{PU} / \mathrm{EES} / \mathrm{KH}-15184$ & $\begin{array}{l}\text { Palearctic \& Nearctic regions; } \\
\text { Indian Himalayas }\end{array}$ \\
\hline Potentilla eriocarpa Wall. ex Lehm. ${ }^{1}$ & $\mathrm{Ph}$ & SA & $2930-2940$ & PU/EES/KH-15186 & Pak. to SW China \\
\hline Potentilla nepalensis Hook. ${ }^{1}$ & $\mathrm{Ph}$ & $\mathrm{BP} / \mathrm{MC}$ & $2260-2790$ & PU/EES/KH-15188 & NE Pak. to W. \& C. Himalayas \\
\hline Prunus cornuta (Wall. ex Royle) Steud. ${ }^{1}$ & DT & MC & $2700-2800$ & PU/EES/KH-1017 & $\begin{array}{l}\text { Himalayas from Afg. to Mya. } \\
\text { \& SW China }\end{array}$ \\
\hline Rosa brunonii Lindl..$^{*}$ & Climbing S & MC & $2580-2600$ & $\mathrm{PU} / \mathrm{EES} / \mathrm{KH}-1208$ & $\begin{array}{l}\text { NE Afg. to China \& Mya., } \\
\text { Himalayas in India }\end{array}$ \\
\hline Rosa webbiana Wall. ex Royle ${ }^{1}$ & DS & $\mathrm{BP}$ & $2310-2400$ & PU/EES/KH-1207 & $\begin{array}{l}\text { C. Asia to W. Himalayas, Tibet } \\
\text { \& Afg. }\end{array}$ \\
\hline Sibbaldia cuneata Schouw ex Kunze ${ }^{1}$ & $\mathrm{Ah}$ & $\mathrm{BP} / \mathrm{MC} / \mathrm{SA}$ & $2200-3810$ & PU/EES/KH-15216 & $\begin{array}{l}\text { Afg. to SW China; Himalayas } \\
\text { in India }\end{array}$ \\
\hline Sorbus lanata (D.Don) S.Schauer ${ }^{1}$ & DT & SA & $3040-3050$ & $\mathrm{PU} / \mathrm{EES} / \mathrm{KH}-1016$ & Afg. to W. Himalayas to Nepal \\
\hline \multicolumn{6}{|l|}{ Rubiaceae } \\
\hline Galium aparine L. $^{1^{*}}$ & Bulbous Ph & $\mathrm{BP} / \mathrm{MC} / \mathrm{SA}$ & $1920-3130$ & PU/EES/KH-15109 & $\begin{array}{l}\text { Europe, N. Africa, Asia minor, } \\
\text { Siberia, Iran, Afg., Pak. \& } \\
\text { Himalayas }\end{array}$ \\
\hline Galium boreale L. $^{1^{*}}$ & Climbing Ah & $\mathrm{BP} / \mathrm{MC} / \mathrm{SA}$ & $2330-3310$ & PU/EES/KH-15110 & $\begin{array}{l}\text { Subarctic \& temp. N. } \\
\text { Hemisphere; throughout India }\end{array}$ \\
\hline \multicolumn{6}{|l|}{ Salicaceae } \\
\hline Populus alba $\mathrm{L}^{2}$ & Dioecious DT & MC & $2430-2440$ & $\mathrm{PU} / \mathrm{EES} / \mathrm{KH}-1014$ & $\begin{array}{l}\text { C. \& S. Europe to Xinjiang \& } \\
\text { W. Himalayas }\end{array}$ \\
\hline Populus ciliata Wall. ex Royle ${ }^{4}$ & Dioecious DT & $\mathrm{BP}$ & $2240-2250$ & $\mathrm{PU} / \mathrm{EES} / \mathrm{KH}-1011$ & $\begin{array}{l}\text { N. Pak. to China \& Mya.; } \\
\text { Himalayas in India }\end{array}$ \\
\hline \multicolumn{6}{|l|}{ Sapindaceae } \\
\hline Acer caesium Wall. ex Brandis ${ }^{2}$ & $\begin{array}{c}\text { Andromonoecious } \\
\text { DT }\end{array}$ & $\mathrm{MC} / \mathrm{SA}$ & $2420-3000$ & $\mathrm{PU} / \mathrm{EES} / \mathrm{KH}-1002$ & $\begin{array}{l}\text { E. Afg. to N. \& EC China; W. } \\
\text { Himalayas in India }\end{array}$ \\
\hline Aesculus indica (Wall. ex Cambess.) Hook. ${ }^{2^{*}}$ & DT & MC & $2750-2800$ & $\mathrm{PU} / \mathrm{EES} / \mathrm{KH}-1003$ & $\begin{array}{l}\text { Afg., Nepal, Pak., E. \& W. } \\
\text { Himalayas }\end{array}$ \\
\hline \multicolumn{6}{|l|}{ Saxifragaceae } \\
\hline Bergenia ligulata Engl. ${ }^{1}$ & $\mathrm{Ph}$ & MC & $2750-2800$ & PU/EES/KH-15038 & $\begin{array}{l}\text { E. Afghanistan to China; } \\
\text { Himalayas in India }\end{array}$ \\
\hline \multicolumn{6}{|l|}{ Scrophulariaceae } \\
\hline Scrophularia decomposita Royle ex Benth. ${ }^{1}$ & $\mathrm{Ph}$ & SA & $2920-3280$ & PU/EES/KH-15209 & $\begin{array}{l}\text { C. Asia; W. Himalayas from } \\
\text { Afg. to Kumaon }\end{array}$ \\
\hline Verbascum thapsus L..$^{*}$ & Prostrate Ah & $\mathrm{MC} / \mathrm{SA}$ & $2620-3150$ & PU/EES/KH-15242 & $\begin{array}{l}\text { Naturalized throughout the } \mathrm{N} \text {. } \\
\text { Hemisphere; Indian Himalayas }\end{array}$ \\
\hline \multicolumn{6}{|l|}{ Solanaceae } \\
\hline Atropa acuminata Royle ex Lindl. ${ }^{5}$ & $\mathrm{Ph}$ & $\mathrm{MC}$ & $2700-2800$ & PU/EES/KH-15037 & Afg., Iran, Pak. \& W. Himalayas \\
\hline Hyoscyamus niger L.. ${ }^{*}$ & $\mathrm{Bh}$ or $\mathrm{Ph}$ & SA & $3140-3150$ & PU/EES/KH-15120 & $\begin{array}{l}\text { Palearctic region; Himalayas } \\
\text { in India }\end{array}$ \\
\hline
\end{tabular}




\begin{tabular}{|c|c|c|c|c|c|}
\hline Family/Taxon & Life-form & Forest type & OER & Voucher no. & Phytogeographic distribution \\
\hline \multicolumn{6}{|l|}{ Taxaceae } \\
\hline Taxus wallichiana Zucc. ${ }^{5}$ & $\begin{array}{c}\text { Dioecious conical } \\
\text { ET }\end{array}$ & MC & $2560-2760$ & $\mathrm{PU} / \mathrm{EES} / \mathrm{KH}-1013$ & $\begin{array}{l}\text { Himalayas from Afg. to SW } \\
\text { China \& Mya. }\end{array}$ \\
\hline \multicolumn{6}{|l|}{ Urticaceae } \\
\hline Urtica dioica $\mathrm{L}^{.{ }^{*}}$ & $\begin{array}{l}\text { Rhizomatous } \\
\text { creeping Ph }\end{array}$ & $\mathrm{BP} / \mathrm{MC} / \mathrm{SA}$ & $2200-3000$ & $\mathrm{PU} / \mathrm{EES} / \mathrm{KH}-15237$ & $\begin{array}{l}\text { Palearctic, introduced in } \\
\text { Neotropic \& Nearctic regions; } \\
\text { throughout India }\end{array}$ \\
\hline \multicolumn{6}{|l|}{ Violaceae } \\
\hline Viola biflora L. $^{1}$ & $\begin{array}{c}\text { Erect } \\
\text { rhizomatous Ph }\end{array}$ & $\mathrm{BP} / \mathrm{MC} / \mathrm{SA}$ & $2200-3120$ & $\mathrm{PU} / \mathrm{EES} / \mathrm{KH}-15245$ & $\begin{array}{l}\text { Palearctic, Mya.; Indian } \\
\text { Himalayas }\end{array}$ \\
\hline Viola canescens Wall. ${ }^{1}$ & $\mathrm{Ph}$ & $\mathrm{BP} / \mathrm{MC} / \mathrm{SA}$ & $2250-2960$ & PU/EES/KH-15246 & $\begin{array}{l}\text { Bhutan, Nepal, India \& Pak.; } \\
\text { Temp. Himalayas \& W. Ghats }\end{array}$ \\
\hline Viola odorata L. $^{1}$ & $\begin{array}{l}\text { Prostrate } \\
\text { rhizomatous Ph }\end{array}$ & $\mathrm{BP} / \mathrm{MC} / \mathrm{SA}$ & $1980-2960$ & $\mathrm{PU} / \mathrm{EES} / \mathrm{KH}-15247$ & $\begin{array}{l}\text { Iran, Iraq, introduced in India } \\
\& \text { Pak. \& Medit. region \& } \\
\text { Caucasia }\end{array}$ \\
\hline Viola pilosa Blume $^{1}$ & $\begin{array}{l}\text { Rhizomatous } \\
\text { prostrate } \mathrm{Ah} \\
\text { or } \mathrm{Ph} \\
\end{array}$ & $\mathrm{BP} / \mathrm{MC} / \mathrm{SA}$ & $1880-2940$ & PU/EES/KH-15248 & $\begin{array}{l}\text { Afg., Pak., Indomalayan; } \\
\text { throughout India }\end{array}$ \\
\hline \multicolumn{6}{|l|}{ Xanthorrhoeaceae } \\
\hline Eremurus himalaicus Baker ${ }^{1}$ & $\mathrm{Ph}$ & SA & $3530-3550$ & PU/EES/KH-15102 & $\begin{array}{l}\text { Afg., Pak. W. Himalayas \& } \\
\text { Tajikistan }\end{array}$ \\
\hline
\end{tabular}

OER-Observed elevation range | 1-Not assessed (NA) | 2-Least Concern (LC) | 3-Critically Endangered (CR) | 4-Data Deficient (DD) | 5-Endangered (EN) | 6-Vulnerable (VU) | S-Shrub | Ph-Perennial herb | Ah-Annual herb | DS-Deciduous shrub | ES-Evergreen shrub | SS-Subshrub | DT-Deciduous tree | ET-Evergreen tree | Bh-Biennial herb |*-Alien species | E-Eastern | S-Southern | N-Northern | W-Western | C-Central | W-Western | SW-Southwestern | SE-Southeastern | NW-North-western | NE-Northeastern | SC-Southcentral | EC-Eastcentral | NC-Northcentral | Afg-Afghanistan | Pak-Pakistan | ThailThailand | Phip-Philippines | Temp-Temperate | Mya-Myanmar | Medit-Mediterranean | Species in bold are endemic to Himalaya.

effects (Qian et al. 2015). The variation in microclimate would have enabled the taxa to adjust to a wide range of niches along elevation and a variety of pre-adapted lineages to colonize in the mountain ranges. Therefore, it can be considered that climatic factors differentiate taxa as indicated by resilience developed over their evolutionary past, with these phylogenetic variations, in turn, deciding species heterogeneity (Wiens \& Donoghue 2004; Rana et al. 2019).

One of the prerequisites for biodiversity conservation is to determine the areas of particular importance in the context of taxa vulnerability and characteristic habitats and critically evaluate the same, thus enabling them to prioritize these areas for further consideration (Spehn 2011). In the present study, the situation for seven (2.57\%) taxa categorized under threatened, i.e., Saussurea costus \& Aconitum chasmanthum (CR), Trillium govanianum, Aconitum heterophyllum, Taxus wallichiana, \& Atropa acuminata (EN), and Cypripedium cordigerum (VU) were found occasionally in the present study and requires immediate conservational priorities across the landscape. Besides climate change and overgrazing, the species in high demand for traditional medicinal and pharmaceutics has led to their extensive collection and illegal trading, thus pushing them closer to extinction (Devi et al. 2014; Nowak et al. 2020). The sustainability of such flora is imperative across the landscape. Ecological rehabilitation, site-specific in particular should be accomplished by re-vegetating degraded sites with natural vegetation. Existing management regulations must be examined in order to adopt strict guidelines to enhance efficiency in decisionmaking and avoid fraud. Extensive quantitative plant diversity inventories and biogeographical explorations ought to be directed on the threatened flora to identify its abundance and frequency. Additionally, ex situ management methods must be in place in addition to the in situ conservation programmes. Overall, from our study we infer that all three types of coniferous forests are rich in flora, demonstrating their importance for conservation. We hope that our results will serve as a benchmark for potential future studies on plant ecology of the area. With notable plant diversity, Kashmir Himalaya is probably a suitable site for further investigations. Moreover, because Kashmir Himalayan forests face threats due to various anthropogenic activities, qualitative data of documented flora will help local and regional authorities to propose management and conservation priorities. 

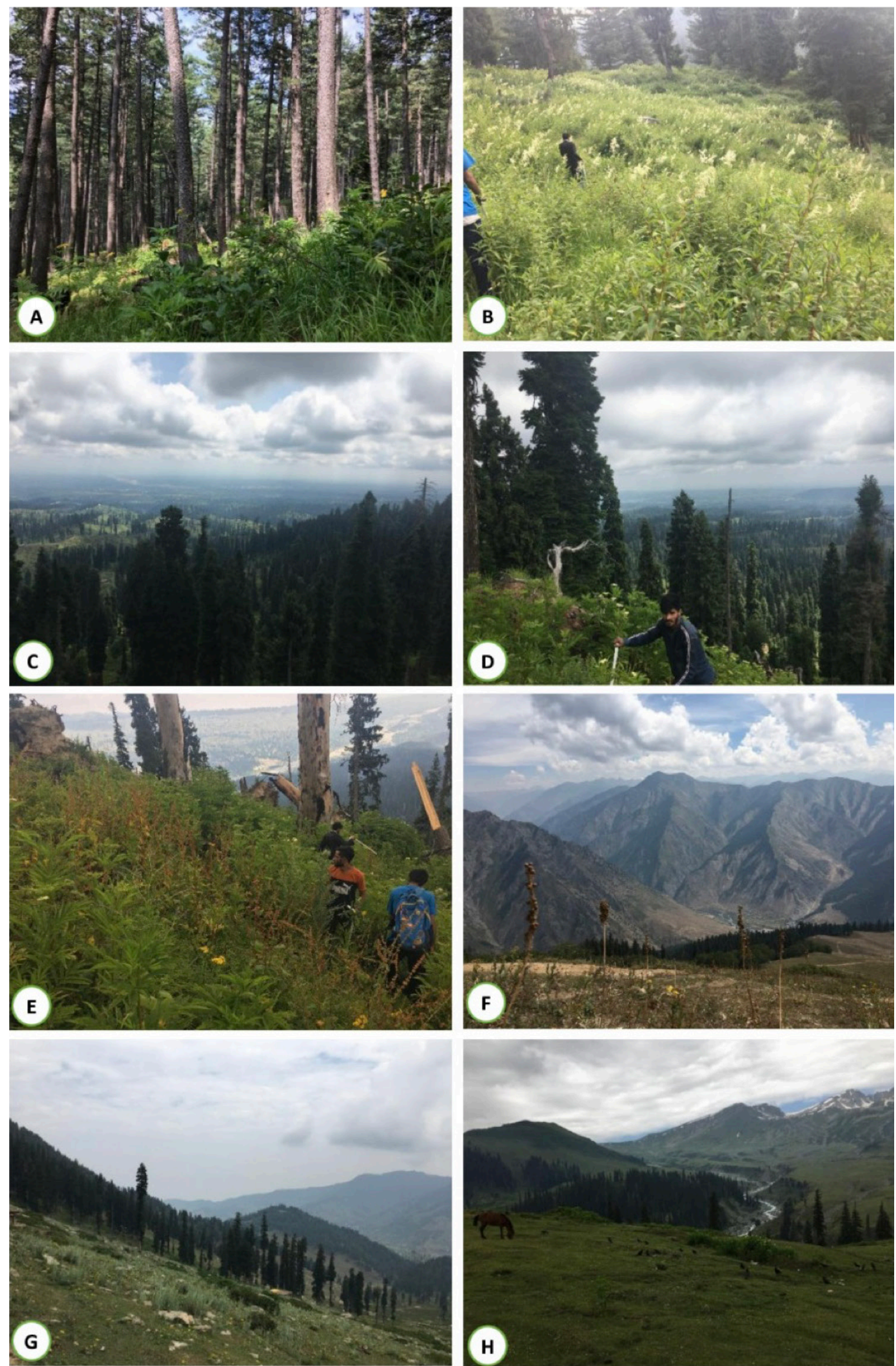

Image 1. Study area overview: A,B-Low-level blue pine forest | C,D,E-Mixed coniferous forest | F,G,H-Sub-alpine forest. @ $\mathrm{C}$ Ashaq Ahmad Dar 

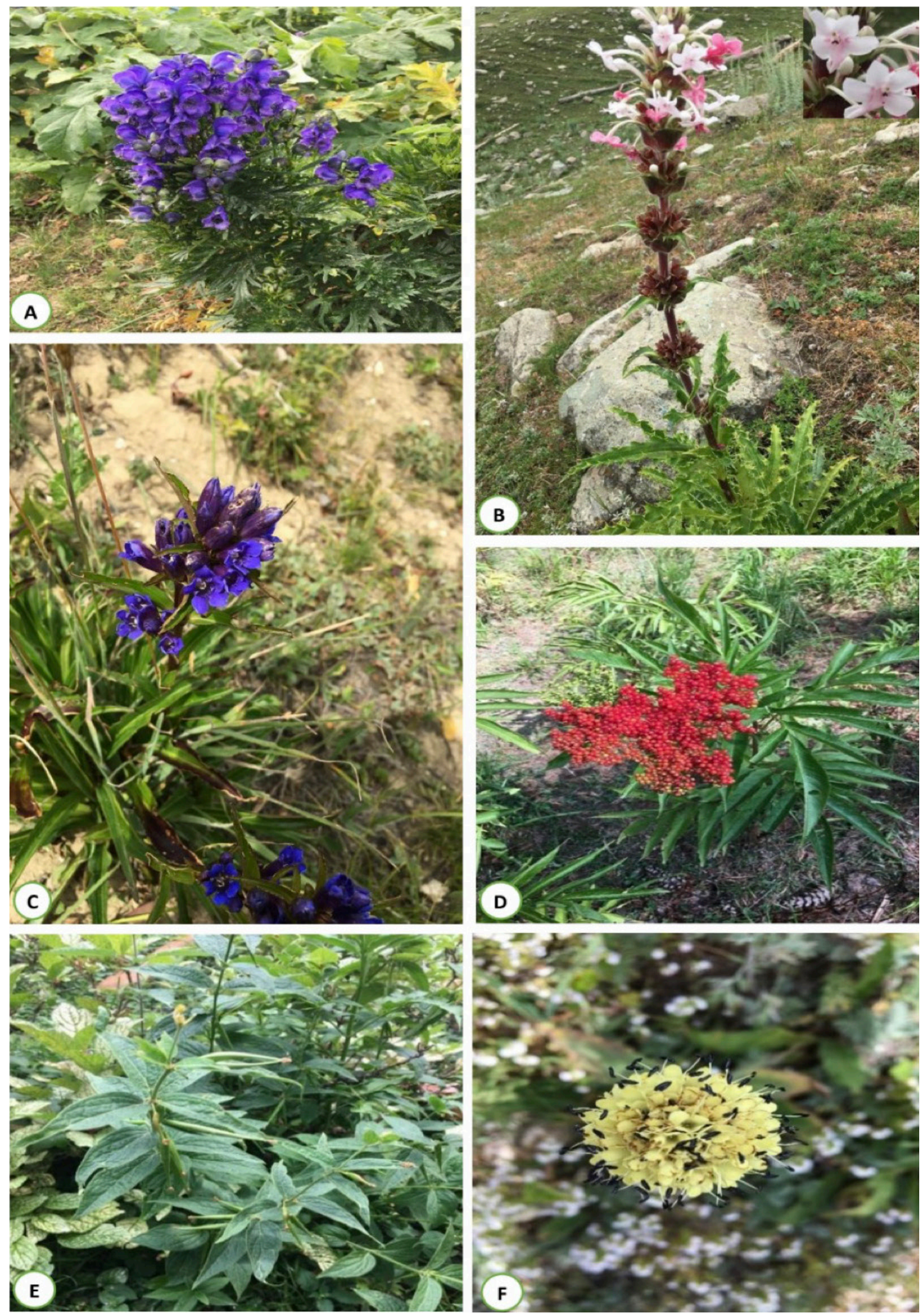

Image 2. Herbs: A-Aconitum chasmanthum | B-Morina longifolia | C-Gentiana tianschanica | D-Sambucus wightiana | E-Vincetoxicum hirundinaria | F-Dipsacus inermis. @) Ashaq Ahmad Dar 

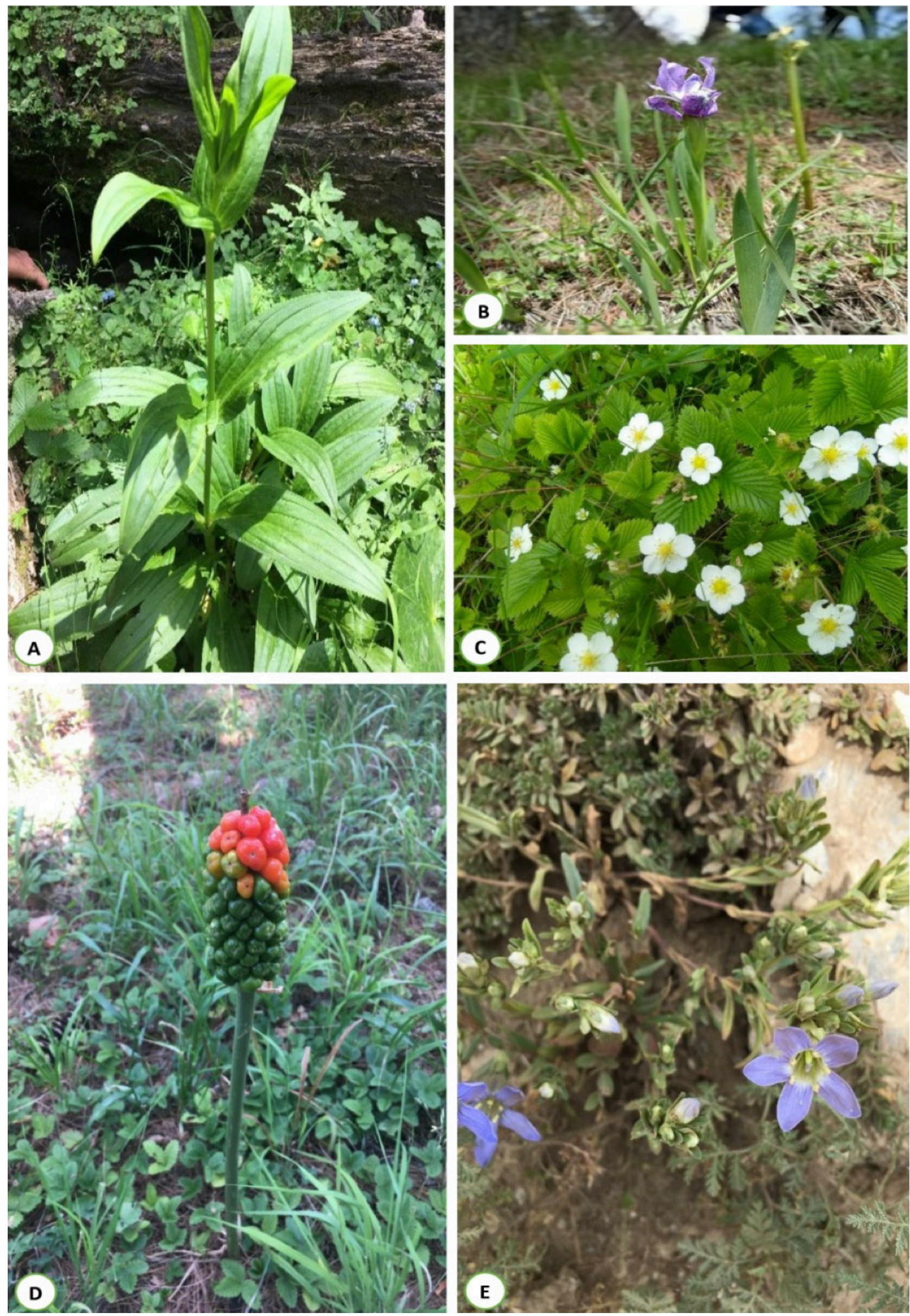

Image 3. Herbs: A-Swertia speciose | B-Iris hookeriana | C-Fragaria nubicola | D-Arisaema jacquemontii | E-Gentiana moorcroftiana. (c) Ashaq Ahmad Dar 

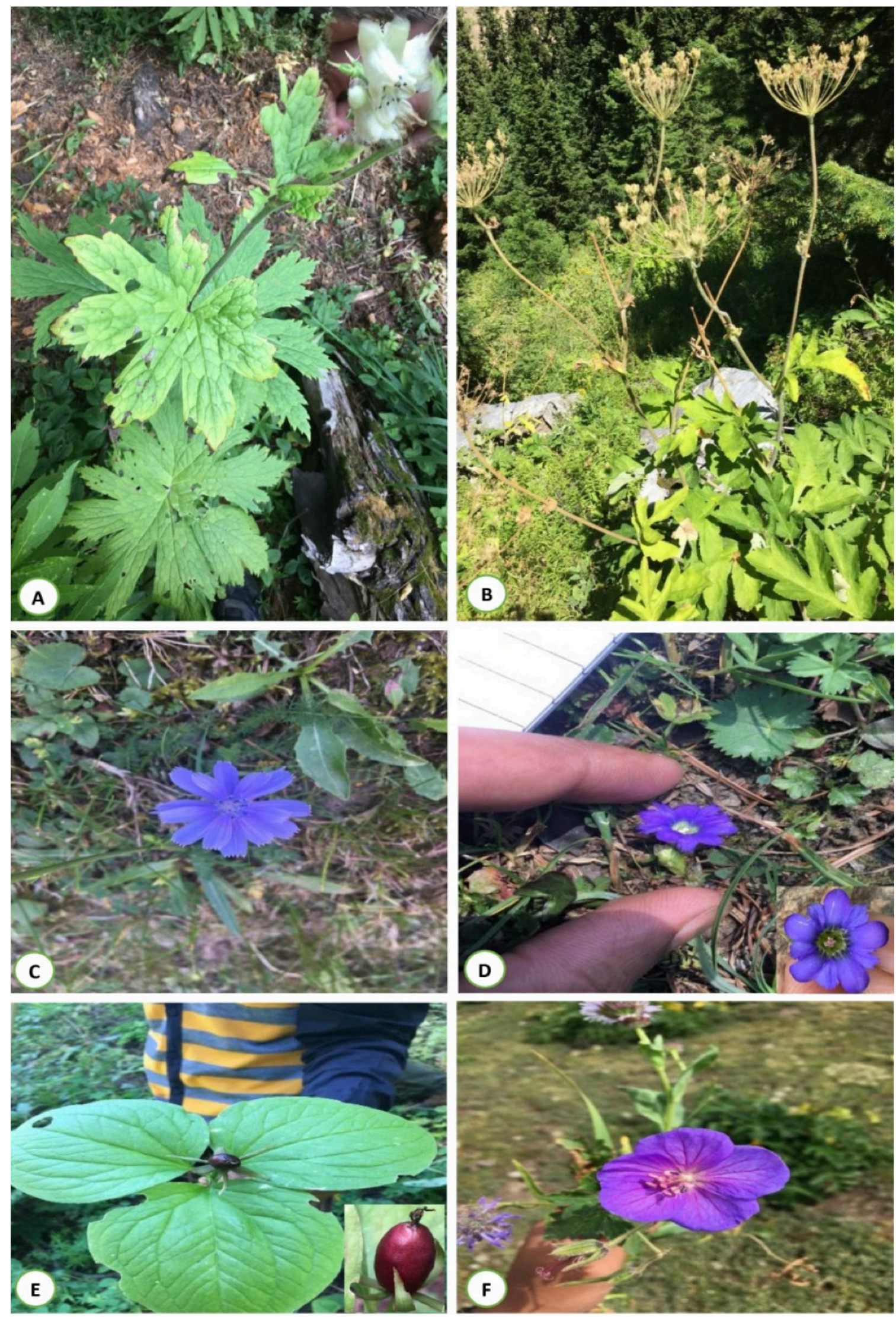

Image 4. Herbs: A-Filipendula vestita | B-Heracleum candicans | C-Cichorium intybus | D-Gentiana carinata | E-Trillium govanianum I E-Geranium wallichianum. @ Ashaq Ahmad Dar 

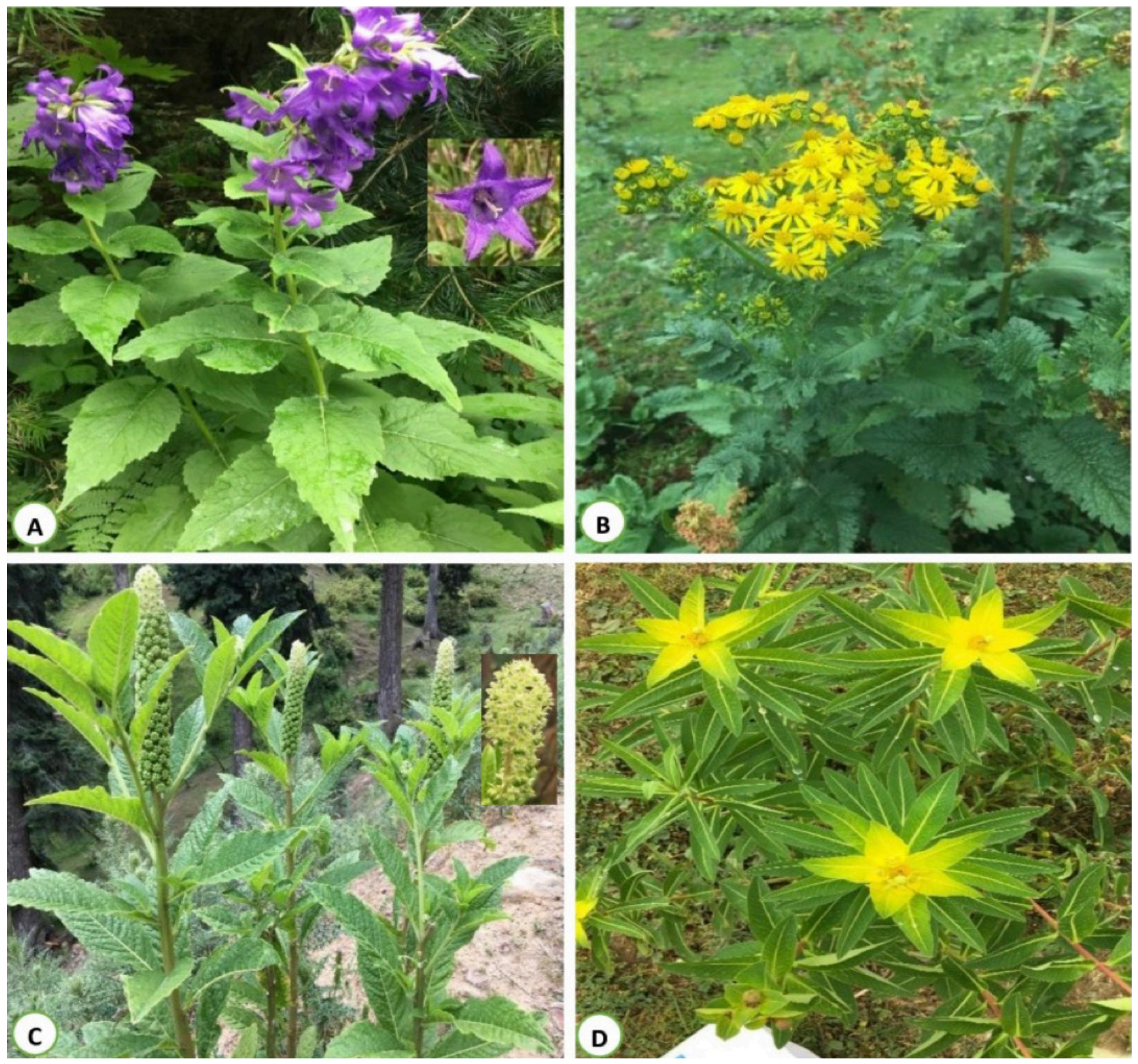

Image 5. Herbs: A-Campanula latifolia | B-Senecio chrysanthemoides | C-Phytolacca acinosa | D-Euphorbia wallichii. (C) Ashaq Ahmad Dar

\section{REFERENCES}

Ahmad, M., S.K. Uniyal, D.R. Batish, H.P. Singh, V. Jaryan, S. Rathee, P. Sharma \& R.K. Kohli (2020). Patterns of plant communities along vertical gradient in Dhauladhar Mountains in Lesser Himalayas in North-Western India. Science of The Total Environment 716: 136919. https://doi.org/10.1016/j.scitotenv.2020.136919

Asif, M., Z. Iqbal, J. Alam, A. Majid, F. Ijaz, N. Ali, I.U. Rahman, S. Hussain, A. Khan \& G. Qadir (2020). Floristic inventory and biological spectra of Balakot, District Mansehra, Pakistan. Acta Ecologica Sinica 40(3): 197-203. https://doi.org/10.1016/j.chnaes.2019.05.009

Bai, F., W. Sang, \& J.C. Axmacher (2011). Forest vegetation responses to climate and environment change: a case study from Changbai Mountain, NE China. Forest Ecology and Management 262: 2052e2060. https://doi.org/10.1016/j.foreco.2011.08.046

Cadotte, M.W. (2006). Dispersal and species diversity: a metaanalysis. American Naturalist 167(6): 913-924. https://doi. org/10.1086/504850

Champion, H.G. \& S.K. Seth (1968). A Revised Survey of the Forest
Types of India (Deli:Government of India Press), 600 pp.

Chase, M.W. \& J.L. Reveal (2009). A phylogenetic classification of the land plants to accompany APG III. Botanical Journal of the Linnean Society 161: 122-127. https://doi.org/10.1111/j.10958339.2009.01002.x

Dad, J.M., M. Muslim, I. Rashid \& Z.A. Reshi (2021). Time series analysis of climate variability and trends in Kashmir Himalaya. Ecological Indicators 126: 107690 . https://doi.org/10.1016/j. ecolind.2021.107690

Dar, J.A. \& S. Sundarapandian (2016). Patterns of plant diversity in seven temperate forest types of Western Himalaya, India. Journal of Asia-Pacific Biodiversity 9: 280-292. https://doi.org/10.1016/j. japb.2016.03.018

Devi, U., P. Sharma, J.C. Rana \& A. Sharma (2014). Phytodiversity assessment in Sangla valley, Northwest Himalaya, India. Check List 10: 740-760. https://doi.org/10.15560/10.4.740

Gairola, S., R.S. Rawal \& U. Dhar (2009). Patterns of litterfall and return of nutrients across anthropogenic disturbance gradients in three subalpine forests of west Himalaya, India. Journal of Forest 

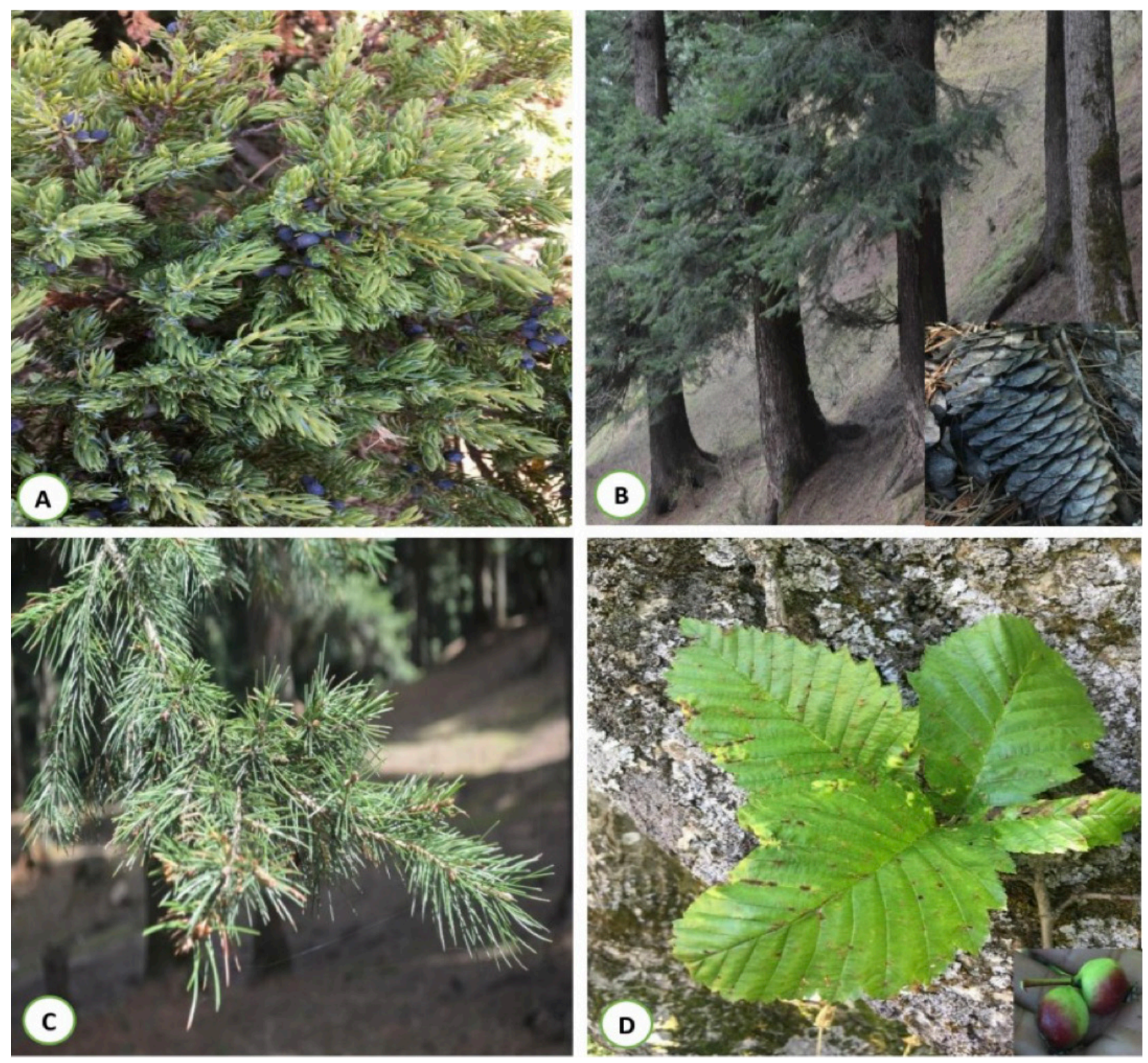

Image 6. Trees: A-Juniperus squamata | B-Abies pindrow | C-Picea smithiana | D-Sorbus lanata. (C) Ashaq Ahmad Dar

Research 14: 73-80. https://doi.org/10.1007/s10310-008-0104-6 Hajra, P.K. (1983). Western Himalayas, pp 49-61. In: Jain S.K. \& A.R.K. Sastry (eds.). Materials for a Catalogue of Threatened Plants of India. BSI, Howrah. 69 pp.

Hooker, J.D. (1872-1897). Flora of British India. Vol. 1 to 7. L. Reeves \& Company, London.

IUCN (2017). Guidelines for using the IUCN Red List categories and criteria. Version 13. Prepared by the Standards and Petitions subcommittee, http://www.iucnredlist.org.

Janssen, J.A.M., J.S. Rodwell, M. Garcia Criado, S. Gubbay, T. Haynes, A. Nieto, N. Sanders, F. Landucci, J. Loidi, A. Ssymank, T. Tahvanainen, M. Valderrabano, A. Acosta, M. Aronsson, G. Arts, F. Attorre, E. Bergmeier, R.-J. Bijlsma, F. Bioret, C. Biţă-Nicolae, I. Biurrun, M. Calix, J. Capelo, A. Čarni, M. Chytry, J. Dengler, P. Dimopoulos, F. Essl, H. Gardfjell, D. Gigante, G. Giusso del Galdo, M. Hajek, F. Jansen, J. Jansen, J. Kapfer, A. Mickolajczak, J.A. Molina, Z. Molnar, D. Paternoster, A. Piernik, B. Poulin, B. Renaux, J.H.J. Schaminee, K. Šumberova, H. Toivonen, T. Tonteri, I. Tsiripidis, R. Tzonev \& M Valachovič (2016). European Red List of Habitats. Part 2: Terrestrial and Freshwater Habitats Publications Office of the European Union, Luxembourg. 44 pp.

Javeid, G.N. (1966). Key to the families of flowering plants of Kashmir Himalaya. Kashmir Science 3(1-2): 101-115.

Javeid, G.N. (1978). Forest flora of Kashmir: a checklist, I. Indian Forester 104(11): 772-779.

Javeid, G.N. (1979). Forest flora of Kashmir: a checklist, II. Indian Forester 105(2): 148-170.

Kachroo, P. (1993). Plant diversity in Northwest Himalaya - a preliminary survey, pp 111-132. In: Dhar, U. (ed.). Himalayan Biodiversity: Conservation Strategies. G.B. Pant Institute of Himalayan Environment and Development, Almora. 553 pp.

Khuroo, A.A., I. Rashid, Z. Reshi, G.H. Dar \& B.A. Wafai (2007). The alien flora of Kashmir Himalaya. Biological Invasions 9: 269-292. https://doi.org/10.1007/s10530-006-9032-6

Körner, C. (2007). The use of altitude in ecological research. Trends in Ecology \& Evolution 22: 569-574. https://doi.org/10.1016/j. tree.2007.09.006

Krishnan, M.S. (1982). Geology of India and Burma, $6^{\text {th }}$ ed. CBS, New 

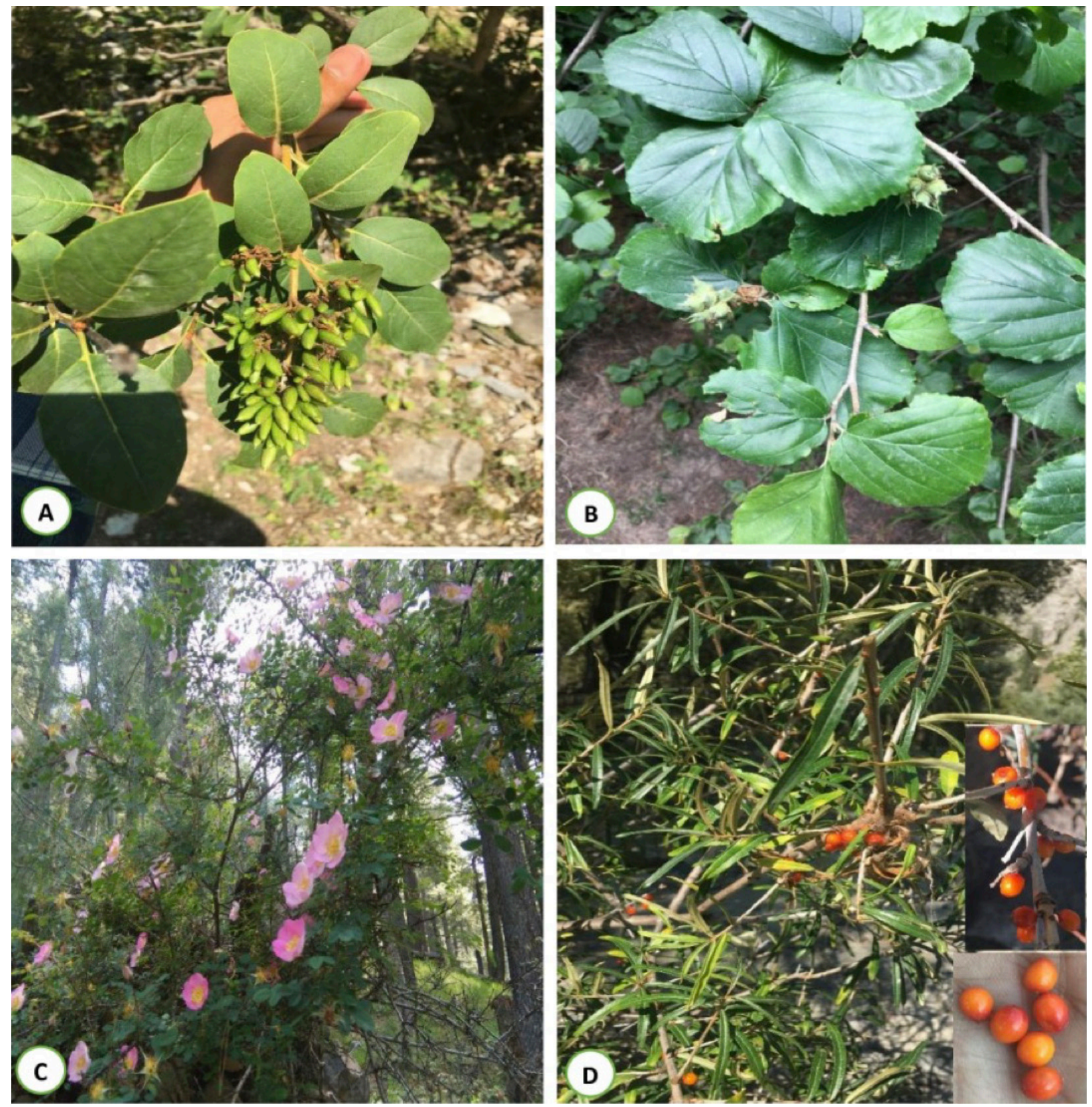

Image 7. Shrubs: A-Syringa emodi | B-Parrotiopsis jacquemontiana | C-Rosa webbiana | D-Hippophae rhamnoides. C Ashaq Ahmad Dar

Delhi, $52 \mathrm{pp}$.

Lambert, W.J. (1933). List of trees and shrubs for the Kashmir \& Jammu forest circles, Jammu \& Kashmir State. Forest Bulletin 80: 1-36.

Maes, D., N.J.B. Isaac, C.A. Harrower, B. Collen, A.J. van Strien \& D.B. Roy (2015). The use of opportunistic data for IUCN Red List assessments. Biological Journal of the Linnean Society 115: 690706. https://doi.org/10.1111/bij.12530

Mahapatra, S.K., C.S. Walia, G.S. Sindhu, K.P.C. Rana \& T. La (2000). Characterization and classification of the soils of different physiographic units in the sub-humid ecosystem of Kashmir region. Journal of the Indian Society of Soil Science 48(3): 572-577.

Malik, A.H., A.A. Khuroo, G.H. Dar \& Z.S. Khan (2010). The woody flora of Jammu \& Kashmir State, India: an updated checklist. Journal of Economic and Taxonomic Botany 34(2): 274-294.

Malik, A.Y., D.P. Singh, M. Yunus, G.A. Bhat, G. Shukla, J. A. Bhat,
M.M. Rather \& S. Chakravarty (2021). Diversity and structure of plant assemblages in open scrub vegetation patches of Dachigam National Park at Kashmir Himalayas. Trees, Forests and People 3: 100060. https://doi.org/10.1016/j.tfp.2020.100060

Mir, N.A., T.H. Masoodi, S.M. Geelani, A.A. Wani, G.N. Parrey \& J.A. Mugloo (2019). Floristic diversity along altitudinal gradient under Betula utilis in North Western Himalayas of Kashmir, India. Acta Ecologica Sinica 39: 362-371. https://doi.org/10.1016/j. chnaes.2019.03.001

Nayar, M.P. (1996). Hot Spots of Endemic Plants of India, Nepal and Bhutan. Tropical Botanic Garden and Research Institute, Thiruvananthapuram, $252 \mathrm{pp}$.

Nowak, A., S. Świerszcz, S. Nowak, H. Hisorev, E. Klichowska, A. Wróbel, A. Nobis \& M. Nobis (2020). Red List of vascular plants of Tajikistan - the core area of the Mountains of Central Asia global 
biodiversity hotspot. Scientific Reports 10: 6235. https://doi. org/10.1038/s41598-020-63333-9

Orsenigo, S., C. Montagnani, G. Fenu, D. Gargano, L. Peruzzi, T. Abeli, A. Alessandrini, G. Bacchetta, F. Bartolucci, M. Bovio, C. Brullo, S. Brullo, A. Carta, M. Castello, D. Cogoni, F. Conti, G. Domina, B. Foggi, M. Gennai, D. Gigante, M. Iberite, C. Lasen, S. Magrini, E. Perrino, F. Prosser, A. Santangelo, A. Selvaggi, A. Stinca, I. Vagge, M. Villani, R. Wagensommer, T. Wilhalm, N. Tartaglini, E. Dupre', C. Blasi \& G. Rossi (2018). Red Listing plants under full national responsibility: extinction risk and threats in the vascular flora endemic to Italy. Biological Conservation 224: 213-222. https://doi. org/10.1016/J.BIOCON.2018.05.030

Peel, M.C., B.L. Finlayson \& T.A. Mcmahon (2007). Updated world map of the Köppen-Geiger climate classification. Hydrology and Earth System Sciences 11: 1633-1644. https://doi.org/10.5194/ hess-11-1633-2007

Polunin, O. \& A. Stainton (1984). Flowers of the Himalaya. Oxford University Press, New Delhi. 580 pp.

Qian, H., J.J. Wiens, J. Zhang \& Y. Zhang (2015). Evolutionary and ecological causes of species richness patterns in North American angiosperm trees. Ecography 38:241-250. https://doi.org/10.1111/ ecog.00952

Rana, S.K., T.D. Price \& H. Qian (2019). Plant species richness across the Himalaya driven by evolutionary history and current climate. Ecosphere 10(11): e02945. https://doi.org/10.1002/ecs2.2945

Rao, R.R. \& B.D. Sharma (1990). A Manual for Herbarium Collection. Botanical Survey of India, Calcutta, 20 pp.

Rashid, I., S.A. Romshoo, R.K. Chaturvedi, N.H. Ravindranath, R. Sukumar, M. Jayaraman, T.V. Lakshmi \& J. Sharma (2015). Projected climate change impacts on vegetation distribution over Kashmir Himalayas. Climatic Change 132: 601-613. https://doi.org/10.1007/ s10584-015-1456-5

Shaheen, H., Z. Ullah, S.M. Khan \& D.M. Harper (2012). Species composition and community structure of western Himalayan moist temperate forests in Kashmir. Forest Ecology Management 278: 138-145. https://doi.org/10.1016/j.foreco.2012.05.009

Sidhu, G.S. \& J.N. Surya (2014). Soils of North-Western Himalayan ecosystem and their land use, constraints, productivity potentials and future strategies. Agropedology 24: 1-19

Singh, J.B. \& P. Kachroo (1994). Forest Flora of Pir Panjal range (Northwestern Himalaya). Bishen Singh Mahendra Pal Singh, Dehradun. 172 pp.

Singh, P., K. Karthigeyan, P. Lakshminarasimhan \& S.S. Dash (2015). Endemic vascular plants of India. Botanical Survey of India, Kolkata. $339 \mathrm{pp}$.

Spehn, E. (2011). Mountain Biodiversity: Effects of Climate Change and How to Manage Them; Sustainable Mountain Development 60, ICIMOD, Kathmandu, Nepal. 40-43 pp.

The Angiosperm Phylogeny Group (2009). An update of the Angiosperm Phylogeny Group classification for the orders and families of flowering plants: APG III. Botanical Journal of the Linnean Society 161: 105-121. https://doi.org/10.1111/boj.12385

The Plant List (2013). Version 1.1. Published on the Internet; http:// www.theplantlist.org/ (accessed 15 January, 2021).

Tiwari, O.P., C.M. Sharma \& Y.S. Rana (2020). Influence of altitude and slope-aspect on diversity, regeneration and structure of some moist temperate forests of Garhwal Himalaya. Tropical Ecology 61: 278-289. https://doi.org/10.1007/s42965-020-00088-4

Wester, P., A. Mishra, A. Mukherji \& A.B. Shrestha (2019). The Hindu Kush Himalaya assessment- mountains, climate change, sustainability and people. Springer Nature, Cham, 627 pp.

Wiens, J.J. \& M.J. Donoghue (2004). Historical biogeogra- phy, ecology, and species richness. Trends in Ecology and Evolution 19: 639-644. https://doi.org/10.1016/j.tree.2004.09.011

Zachos, F.E. \& J.C. Habel (2011). Biodiversity hotspots: distribution and protection of conservation priority areas. Springer, Berlin/ Heidelberg, 565 pp. 

Dr. John Noyes, Natural History Museum, London, UK

Dr. Albert G. Orr, Griffith University, Nathan, Australia

Dr. Sameer Padhye, Katholieke Universiteit Leuven, Belgium

Dr. Nancy van der Poorten, Toronto, Canada

Dr. Kareen Schnabel, NIWA, Wellington, New Zealand

Dr. R.M. Sharma, (Retd.) Scientist, Zoological Survey of India, Pune, India

Dr. Manju Siliwal, WILD, Coimbatore, Tamil Nadu, India

Dr. G.P. Sinha, Botanical Survey of India, Allahabad, India

Dr. K.A. Subramanian, Zoological Survey of India, New Alipore, Kolkata, India

Dr. P.M. Sureshan, Zoological Survey of India, Kozhikode, Kerala, India

Dr. R. Varatharajan, Manipur University, Imphal, Manipur, India

Dr. Eduard Vives, Museu de Ciències Naturals de Barcelona, Terrassa, Spain

Dr. James Young, Hong Kong Lepidopterists' Society, Hong Kong

Dr. R. Sundararaj, Institute of Wood Science \& Technology, Bengaluru, India

Dr. M. Nithyanandan, Environmental Department, La Ala Al Kuwait Real Estate. Co. K.S.C.,

Kuwait

Dr. Himender Bharti, Punjabi University, Punjab, India

Mr. Purnendu Roy, London, UK

Dr. Saito Motoki, The Butterfly Society of Japan, Tokyo, Japan

Dr. Sanjay Sondhi, TITLI TRUST, Kalpavriksh, Dehradun, India

Dr. Nguyen Thi Phuong Lien, Vietnam Academy of Science and Technology, Hanoi, Vietnam

Dr. Nitin Kulkarni, Tropical Research Institute, Jabalpur, India

Dr. Robin Wen Jiang Ngiam, National Parks Board, Singapore

Dr. Lional Monod, Natural History Museum of Geneva, Genève, Switzerland.

Dr. Asheesh Shivam, Nehru Gram Bharti University, Allahabad, India

Dr. Rosana Moreira da Rocha, Universidade Federal do Paraná, Curitiba, Brasi

Dr. Kurt R. Arnold, North Dakota State University, Saxony, Germany

Dr. James M. Carpenter, American Museum of Natural History, New York, USA

Dr. David M. Claborn, Missouri State University, Springfield, USA

Dr. Kareen Schnabel, Marine Biologist, Wellington, New Zealand

Dr. Amazonas Chagas Júnior, Universidade Federal de Mato Grosso, Cuiabá, Brasil

Mr. Monsoon Jyoti Gogoi, Assam University, Silchar, Assam, India

Dr. Heo Chong Chin, Universiti Teknologi MARA (UiTM), Selangor, Malaysia

Dr. R.J. Shiel, University of Adelaide, SA 5005, Australia

Dr. Siddharth Kulkarni, The George Washington University, Washington, USA

Dr. Priyadarsanan Dharma Rajan, ATREE, Bengaluru, India

Dr. Phil Alderslade, CSIRO Marine And Atmospheric Research, Hobart, Australia

Dr. John E.N. Veron, Coral Reef Research, Townsville, Australia

Dr. Daniel Whitmore, State Museum of Natural History Stuttgart, Rosenstein, Germany.

Dr. Yu-Feng Hsu, National Taiwan Normal University, Taipei City, Taiwan

Dr. Keith V. Wolfe, Antioch, California, USA

Dr. Siddharth Kulkarni, The Hormiga Lab, The George Washington University, Washington,

D.C., USA

Dr. Tomas Ditrich, Faculty of Education, University of South Bohemia in Ceske

Budejovice, Czech Republic

Dr. Mihaly Foldvari, Natural History Museum, University of Oslo, Norway

Dr. V.P. Uniyal, Wildlife Institute of India, Dehradun, Uttarakhand 248001, India

Dr. John T.D. Caleb, Zoological Survey of India, Kolkata, West Bengal, India

Dr. Priyadarsanan Dharma Rajan, Ashoka Trust for Research in Ecology and the Environment

(ATREE), Royal Enclave, Bangalore, Karnataka, India

\section{Fishes}

Dr. Neelesh Dahanukar, IISER, Pune, Maharashtra, India

Dr. Topiltzin Contreras MacBeath, Universidad Autónoma del estado de Morelos, México

Dr. Heok Hee Ng, National University of Singapore, Science Drive, Singapore

Dr. Rajeev Raghavan, St. Albert's College, Kochi, Kerala, India

Dr. Robert D. Sluka, Chiltern Gateway Project, A Rocha UK, Southall, Middlesex, UK

Dr. E. Vivekanandan, Central Marine Fisheries Research Institute, Chennai, India

Dr. Davor Zanella, University of Zagreb, Zagreb, Croatia

Dr. A. Biju Kumar, University of Kerala, Thiruvananthapuram, Kerala, India

Dr. Akhilesh K.V., ICAR-Central Marine Fisheries Research Institute, Mumbai Research

Centre, Mumbai, Maharashtra, India

Dr. J.A. Johnson, Wildlife Institute of India, Dehradun, Uttarakhand, India

Amphibians

Dr. Sushil K. Dutta, Indian Institute of Science, Bengaluru, Karnataka, India

Dr. Annemarie Ohler, Muséum national d'Histoire naturelle, Paris, France

\section{Reptiles}

Dr. Gernot Vogel, Heidelberg, Germany

Dr. Raju Vyas, Vadodara, Gujarat, India

Dr. Pritpal S. Soorae, Environment Agency, Abu Dubai, UAE.

Prof. Dr. Wayne J. Fuller, Near East University, Mersin, Turkey

Prof. Chandrashekher U. Rivonker, Goa University, Taleigao Plateau, Goa. India

Dr. S.R. Ganesh, Chennai Snake Park, Chennai, Tamil Nadu, India

Dr. Himansu Sekhar Das, Terrestrial \& Marine Biodiversity, Abu Dhabi, UAE
Birds

Dr. Hem Sagar Baral, Charles Sturt University, NSW Australia

Dr. Chris Bowden, Royal Society for the Protection of Birds, Sandy, UK

Dr. Priya Davidar, Pondicherry University, Kalapet, Puducherry, India

Dr. J.W. Duckworth, IUCN SSC, Bath, UK

Dr. Rajah Jayapal, SACON, Coimbatore, Tamil Nadu, India

Dr. Rajiv S. Kalsi, M.L.N. College, Yamuna Nagar, Haryana, India

Dr. V. Santharam, Rishi Valley Education Centre, Chittoor Dt., Andhra Pradesh, India

Dr. S. Balachandran, Bombay Natural History Society, Mumbai, India

Mr. J. Praveen, Bengaluru, India

Dr. C. Srinivasulu, Osmania University, Hyderabad, India

Dr. K.S. Gopi Sundar, International Crane Foundation, Baraboo, USA

Dr. Gombobaatar Sundev, Professor of Ornithology, Ulaanbaatar, Mongolia

Prof. Reuven Yosef, International Birding \& Research Centre, Eilat, Israel

Dr. Taej Mundkur, Wetlands International, Wageningen, The Netherlands

Dr. Carol Inskipp, Bishop Auckland Co., Durham, UK

Dr. Tim Inskipp, Bishop Auckland Co, Durham, UK

Dr. V. Gokula, National College, Tiruchirappalli, Tamil Nadu, India

Dr. Arkady Lelej, Russian Academy of Sciences, Vladivostok, Russia

Dr. Simon Dowell, Science Director, Chester Zoo, UK

Dr. Mário Gabriel Santiago dos Santos, Universidade de Trás-os-Montes e Alto Douro,

Quinta de Prados, Vila Real, Portugal

Dr. Grant Connette, Smithsonian Institution, Royal, VA, USA

Dr. M. Zafar-ul Islam, Prince Saud Al Faisal Wildlife Research Center, Taif, Saudi Arabia

Mammals

Dr. Giovanni Amori, CNR - Institute of Ecosystem Studies, Rome, Italy

Dr. Anwaruddin Chowdhury, Guwahati, India

Dr. David Mallon, Zoological Society of London, UK

Dr. Shomita Mukherjee, SACON, Coimbatore, Tamil Nadu, India

Dr. Angie Appel, Wild Cat Network, Germany

Dr. P.O. Nameer, Kerala Agricultural University, Thrissur, Kerala, India

Dr. Ian Redmond, UNEP Convention on Migratory Species, Lansdown, UK

Dr. Heidi S. Riddle, Riddle's Elephant and Wildlife Sanctuary, Arkansas, USA

Dr. Karin Schwartz, George Mason University, Fairfax, Virginia.

Dr. Lala A.K. Singh, Bhubaneswar, Orissa, India

Dr. Mewa Singh, Mysore University, Mysore, India

Dr. Paul Racey, University of Exeter, Devon, UK

Dr. Honnavalli N. Kumara, SACON, Anaikatty P.O., Coimbatore, Tamil Nadu, India

Dr. Nishith Dharaiya, HNG University, Patan, Gujarat, India

Dr. Spartaco Gippoliti, Socio Onorario Società Italiana per la Storia della Fauna "Giuseppe

Altobello", Rome, Italy

Dr. Justus Joshua, Green Future Foundation, Tiruchirapalli, Tamil Nadu, India

Dr. H. Raghuram, The American College, Madurai, Tamil Nadu, India

Dr. Paul Bates, Harison Institute, Kent, UK

Dr. Jim Sanderson, Small Wild Cat Conservation Foundation, Hartford, USA

Dr. Dan Challender, University of Kent, Canterbury, UK

Dr. David Mallon, Manchester Metropolitan University, Derbyshire, UK

Dr. Brian L. Cypher, California State University-Stanislaus, Bakersfield, CA

Dr. S.S. Talmale, Zoological Survey of India, Pune, Maharashtra, India

Prof. Karan Bahadur Shah, Budhanilakantha Municipality, Kathmandu, Nepal

Dr. Susan Cheyne, Borneo Nature Foundation International, Palangkaraja, Indonesia

Dr. Hemanta Kafley, Wildlife Sciences, Tarleton State University, Texas, USA

\section{Other Disciplines}

Dr. Aniruddha Belsare, Columbia MO 65203, USA (Veterinary)

Dr. Mandar S. Paingankar, University of Pune, Pune, Maharashtra, India (Molecular)

Dr. Jack Tordoff, Critical Ecosystem Partnership Fund, Arlington, USA (Communities)

Dr. Ulrike Streicher, University of Oregon, Eugene, USA (Veterinary)

Dr. Hari Balasubramanian, EcoAdvisors, Nova Scotia, Canada (Communities)

Dr. Rayanna Hellem Santos Bezerra, Universidade Federal de Sergipe, São Cristóvão, Brazil

Dr. Jamie R. Wood, Landcare Research, Canterbury, New Zealand

Dr. Wendy Collinson-Jonker, Endangered Wildlife Trust, Gauteng, South Africa

Dr. Rajeshkumar G. Jani, Anand Agricultural University, Anand, Gujarat, India

Dr. O.N. Tiwari, Senior Scientist, ICAR-Indian Agricultural Research Institute (IARI), New

Delhi, India

Dr. L.D. Singla, Guru Angad Dev Veterinary and Animal Sciences University, Ludhiana, India

Dr. Rupika S. Rajakaruna, University of Peradeniya, Peradeniya, Sri Lanka

Dr. Bahar Baviskar, Wild-CER, Nagpur, Maharashtra 440013, India

Reviewers 2018-2020

Due to pausity of space, the list of reviewers for $2018-2020$ is available online.

The opinions expressed by the authors do not reflect the views of the Journal of Threatened Taxa, Wildlife Information Liaison Development Society, Zoo Outreach Organization, or any of the partners. The journal, the publisher, the host, and the partners are not responsible for the accuracy of the political boundaries shown in the maps by the authors.

Journal of Threatened Taxa is indexed/abstracted in Bibliography of Systematic Mycology, Biological Abstracts, BIOSIS Previews, CAB Abstracts, EBSCO, Google Scholar, Index Copernicus, Index Fungorum, JournalSeek, National Academy of Agricultural Sciences, NewJour, OCLC WorldCat, SCOPUS, Stanford University Libraries, Virtual Library of Biology, Zoological Records.

NAAS rating (India) 5.64
Print copies of the Journal are available at cost. Write to:

The Managing Editor, JoTT,

c/o Wildlife Information Liaison Development Society,

No. 12, Thiruvannamalai Nagar, Saravanampatti - Kalapatti Road,

Saravanampatti, Coimbatore, Tamil Nadu 641035, India

ravi@threatenedtaxa.org 


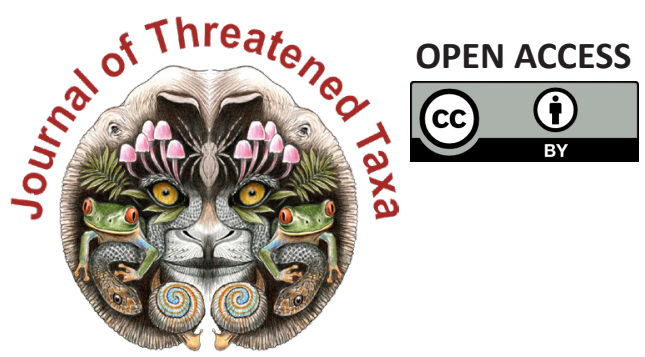

www.threatenedtaxa.org

The Journal of Threatened Taxa (JoTT) is dedicated to building evidence for conservation globally by publishing peer-reviewed articles online every month at a reasonably rapid rate at www.threatenedtaxa.org. All articles published in JoTT are registered under Creative Commons Attribution 4.0 International License unless otherwise mentioned. JoTT allows allows unrestricted use, reproduction, and distribution of articles in any medium by providing adequate credit to the author(s) and the source of publication.

\section{ISSN $0974-7907$ (Online) | ISSN $0974-7893$ (Print)}

\section{January 2022 | Vol. 14 | No. 1 | Pages: 20311-20538 \\ Date of Publication: 26 January 2022 (Online \& Print) DOI: 10.11609/jott.2022.14.1.20311-20538}

Articles

Estimating the completeness of orchid checklists and atlases: a case study from southern Italy

- Antonio Croce, Pp. 20311-20322

A floristic survey across three coniferous forests of Kashmir Himalaya, India - a checklist

- Ashaq Ahmad Dar, Akhtar Hussain Malik \& Narayanaswamy Parthasarathy, Pp. 20323-20345

Associations of butterflies across different forest types in Uttarakhand, western Himalaya, India: implications for conservation planning

- Arun Pratap Singh, Pp. 20346-20370

Comparison of bird diversity in protected and non-protected wetlands of western lowland of Nepal

- Jagan Nath Adhikari, Janak Raj Khatiwada, Dipendra Adhikari, Suman Sapkota, Bishnu Prasad Bhattarai, Deepak Rijal \& Lila Nath Sharma, Pp. 20371-20386

Local hunting practices and perceptions regarding the distribution and ecological role of the Large Flying Fox (Chiroptera: Pteropodidae: Pteropus vampyrus) in western Sarawak, Malaysian Borneo

- Jayasilan Mohd-Azlan, Joon Yee Yong, Nabila Norshuhadah Mohd Hazzrol, Philovenny Pengiran, Arianti Atong \& Sheema Abdul Aziz, Pp. 20387-20399

\section{Communications}

Macrolichens of Mathikettan Shola National Park, Western Ghats: a preliminary investigation with some new records

- Aswathi Anilkumar, Stephen Sequeira, Arun Christy \& S.M. Arsha, Pp. 20400-20405

New distribution record of globally threatened Ocean Turf Grass Halophila beccarii Ascherson, 1871 from the North Andaman Islands highlights the importance of seagrass exploratory surveys

- Swapnali Gole, Prasad Gaidhani, Srabani Bose, Anant Pande, Jeyaraj Antony Johnson \& Kuppusamy Sivakumar, Pp. 20406-20412

An inventory of new orchid (Orchidaceae) records from Kozhikode, Kerala, India - M. Sulaiman, C. Murugan \& M.U. Sharief, Pp. 20413-20425

Abundance and spatial distribution analyses of Stemonoporus moonii Thwaites (Dipterocarpaceae) - a critically endangered species endemic to Sri Lanka - K.A.M.R.P. Atapattu, H.D.D.C.K. Perera, H.S. Kathriarachchi \& A.R. Gunawardena, Pp. 20426-20432

Plant diversity of Point Calimere Wildlife Sanctuary and fodder species grazed by the Blackbuck Antilope cervicapra L.

- Ashutosh Kumar Upadhyay, A. Andrew Emmanuel, Ansa Sarah Varghese \&

D. Narasimhan, Pp. 20433-20443

Raptors observed (1983-2016) in National Chambal Gharial Sanctuary: semi-arid biogeographic region suggestions for parametric studies on ecological continuity in Khathiar-Gir Ecoregion, India

- L.A.K. Singh, R.K. Sharma \& Udayan Rao Pawar, Pp. 20444-20460

Nesting success of Sharpe's Longclaw (Macronyx sharpei Jackson, 1904) around the grasslands of lake Ol'bolossat Nyandarua, Kenya

- Hamisi Ann Risper, Charles M. Warui \& Peter Njoroge, Pp. 20461-20468

Population, distribution and diet composition of Smooth-coated Otter Lutrogale perspicillata Geoffroy, 1826 in Hosur and Dharmapuri Forest Divisions, India - Nagarajan Baskaran, Raman Sivaraj Sundarraj \& Raveendranathanpillai Sanil, Pp. 20469-20477

Utilization of home garden crops by primates and current status of human-primate interface at Galigamuwa Divisional Secretariat Division in Kegalle District, Sri Lanka

- Charmalie Anuradhie Dona Nahallage, Dahanakge Ayesha Madushani Dasanayake, Dilan Thisaru Hewamanna \& Dissanayakalage Tharaka Harshani Ananda, Pp. 2047820487
Revival of Eastern Swamp Deer Rucervus duvaucelii ranjitsinhi (Groves, 1982) in Manas National Park of Assam, India

- Nazrul Islam, Aftab Ahmed, Rathin Barman, Sanatan Deka, Bhaskar Choudhury, Prasanta Kumar Saikia \& Jyotishman Deka, Pp. 20488-20493

Trypanosoma evansi infection in a captive Indian Wolf Canis lupus pallipes - molecular diagnosis and therapy

- Manojita Dash, Sarat Kumar Sahu, Santosh Kumar Gupta, Niranjana Sahoo \& Debarat Mohapatra, Pp. 20494-20499

View Point

COVID-19 and civil unrest undoing steady gains in karst conservation and herpetological research in Myanmar, and an impediment to progress - Evan S.H. Quah, Lee L. Grismer, Perry L. Wood, Jr., Aung Lin \& Myint Kyaw Thura, Pp. 20500-20502

\section{Short Communications}

Morphological characterization and mt DNA barcode of a tiger moth species, Asota ficus (Fabricius, 1775) (Lepidoptera: Noctuoidea: Erebidae: Aganainae) from India - Aparna Sureshchandra Kalawate, K.P. Dinesh \& A. Shabnam, Pp. 20503-20510

Distribution of Smooth-coated Otters Lutrogale perspicillata (Mammalia: Carnivora: Mustelidae): in Ratnagiri, Maharashtra, India

- Swanand Patil \& Kranti Yardi, Pp. 20511-20516

Wildlife at the crossroads: wild animal road kills due to vehicular collision on a mountainous highway in northwestern Himalayan region

- Muzaffar A. Kichloo, Asha Sohil \& Neeraj Sharma, Pp. 20517-20522

Notes

Robiquetia gracilis (Lindl.) Garay-a new record to the flora of Anamalai Hills, Tami Nadu, India

- B. Subbaiyan, V. Ganesan, P.R. Nimal Kumar \& S. Thangaraj Panneerselvam, Pp. 20523-20525

Ipomoea laxiflora H.J. Chowdhery \& Debta (Convolvulaceae): new records for the Western Ghats and semiarid regions

- Sachin M. Patil, Ajit M. Vasava, Vinay M. Raole \& Kishore S. Rajput, Pp. 20526-20529

Counting the cost: high demand puts Bunium persicum (Boiss.) B.Fedtsch. in jeopardy

- Monika Sharma, Manisha Mathela, Rupali Sharma, Himanshu Bargali, Gurinderjit S Goraya \& Amit Kumar, Pp. 20530-20533

First record of Parasitic Jaeger Stercorarius parasiticus (Aves: Charadriiformes: Stercorariidae) from inland freshwater Inle Lake, Myanmar

- Sai Sein Lin Oo, Myint Kyaw, L.C.K. Yun, Min Zaw Tun, Yar Zar Lay Naung, Soe Naing Aye \& Swen C. Renner, Pp. 20534-20536

\section{Book Review}

Capparis of India

- V. Sampath Kumar, Pp. 20537-20538
Publisher \& Host
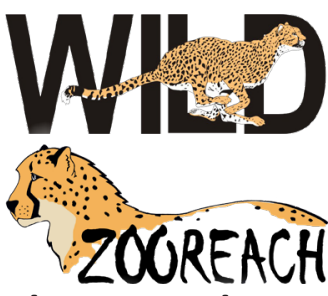

Threatened Taxa 